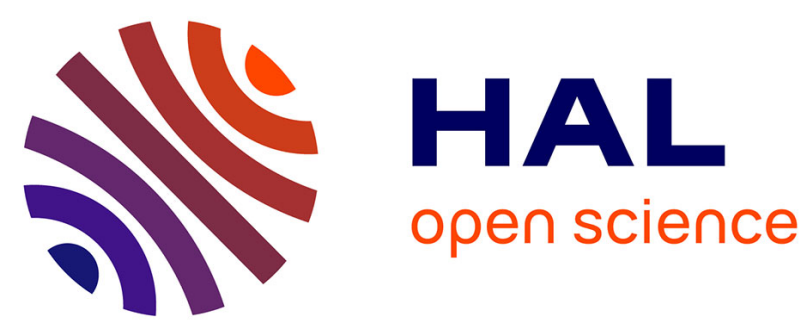

\title{
Turbulent impinging jet flow into an unshrouded rotor-stator system: Hydrodynamics and heat transfer
}

Sébastien Poncet, Thien Duy Nguyen, Souad Harmand, Julien Pellé, Riccardo da Soghe, Cosimo Bianchini, Stéphane Viazzo

\section{- To cite this version:}

Sébastien Poncet, Thien Duy Nguyen, Souad Harmand, Julien Pellé, Riccardo da Soghe, et al.. Turbulent impinging jet flow into an unshrouded rotor-stator system: Hydrodynamics and heat transfer. International Journal of Heat and Fluid Flow, 2013, 44, pp.719-734. 10.1016/j.ijheatfluidflow.2013.10.001 . hal-00976669

\section{HAL Id: hal-00976669 https://hal.science/hal-00976669}

Submitted on 10 Apr 2014

HAL is a multi-disciplinary open access archive for the deposit and dissemination of scientific research documents, whether they are published or not. The documents may come from teaching and research institutions in France or abroad, or from public or private research centers.
L'archive ouverte pluridisciplinaire HAL, est destinée au dépôt et à la diffusion de documents scientifiques de niveau recherche, publiés ou non, émanant des établissements d'enseignement et de recherche français ou étrangers, des laboratoires publics ou privés. 


\title{
Turbulent impinging jet flow into an unshrouded rotor-stator system: hydrodynamics and heat transfer
}

\author{
Sébastien Poncet, ${ }^{1}$ Stéphane Viazzo \\ Laboratoire M2P2, UMR 7340 CNRS - Aix-Marseille Université, IMT la Jetée, 38 \\ rue Joliot-Curie, 13451 Marseille (France) \\ Thien Duy Nguyen, Souad Harmand, Julien Pellé \\ Laboratoire TEMPO - D2FT, Universités de Valenciennes et de Lille Nord de \\ France, 59313 Valenciennes (France) \\ Riccardo Da Soghe, Cosimo Bianchini \\ Dipartimento di Energetica "Sergio Stecco", Università degli Studi di Firenze, Via \\ S.Marta 3, 50139 Firenze (Italy)
}

\begin{abstract}
New calculations using an innovative Reynolds Stress Model are compared to velocity measurements performed by Particle Image Velocimetry technique and the predictions of a $k-\omega$ SST model in the case of an impinging jet flow onto a rotating disk in a discoidal and unshrouded rotor-stator system. The cavity is characterized by a dimensionless spacing interval $G=0.02$ and a low aspect ratio for the jet $e / D=0.25$. Jet Reynolds numbers ranging from $1.72 \times 10^{4}$ to $4.3 \times 10^{4}$ and rotational Reynolds numbers between $0.33 \times 10^{5}$ and $5.32 \times 10^{5}$ are considered. Three flow regions have been identified: a jet-dominated flow area at low radii characterized by a zero tangential velocity, a mixed region at intermediate radii and rotationdominated flow region outwards. For all parameters, turbulence, which tends to the isotropic limit in the core, is much intense in a region located after the impingement zone. A relative good agreement between the PIV measurements and the predictions of the RSM has been obtained in terms of the radial distributions of the core-swirl ratio and of the turbulence intensities. The $k-\omega$ SST model overstimates these flow characteristics in the jet dominated area. For the thermal field, the heat transfers are enhanced in the jet dominated region and decreases towards the periphery of the cavity. The jet Reynolds number appears to have a preponderant effect compared to the rotational one on the heat transfer distribution. The two RANS modelings compare quite well with the heat transfer measurements for these ranges of parameters.
\end{abstract}


Key words: Rotor-stator, impinging jet, heat transfer, RANS modeling, Particle Image Velocimetry, Infrared thermography

\section{Introduction}

Rotating disk configurations are often encountered in the turbomachinery industry for gas turbine engines or hard-disk drives as examples. In such complex systems, an effective cooling system is nearly always required to remove heat due to the high rotation rates reached in very small clearances. The specific engineering application of this study is an alternator, which can be found in a wind generator $(3 \mathrm{~m}$ radius, maximum rotational speed of $25 \mathrm{rpm}$, delivered power of $750 \mathrm{~kW}$ ) established in the north of France. It consists of a discoidal rotor-stator system, which does not use gears allowing the generators to operate at low rotational speeds while reducing energy losses. The main technological lock consists in solving the ineffective cooling due to high electrical losses dissipated for a relative low rotational speed. An improvement on the cooling of discoidal rotor-stator alternators could be obtained by using air jet impingement. In this paper, comparisons between velocity and temperature measurements and the new predictions of both the Reynolds Stress Model of Elena and Schiestel [1] and a $k-\omega$ SST model are thus provided for an open air-gap rotor-stator cavity with an axial impinging jet.

From a global point of view, when a stationary disk (without any opening and jet) faces a rotating one, a centrifugal flow occurs near the rotor while a centripetal flow develops near the stator. Depending on the aspect ratio of the cavity $G=e / R_{e x t}$ ( $e$ the interdisk spacing and $R_{e x t}$ the rotor radius), the rotational Reynolds number $R e=\omega R_{e x t}^{2} / \nu$ ( $\omega$ the rotation rate of the rotor and $\nu$ the kinematic viscosity of the fluid) and the type of rotor-stator configuration (shrouded or not), four flow regimes may be observed. On one hand, it can be either laminar or turbulent. On the other hand, the two boundary layers can be merged or not. This classification has been firstly introduced by Daily and Nece [2] in the case of a shrouded cavity and then by Pellé and Harmand [3] for an unshrouded one. When both boundary layers are merged, the flow is characterized by strong viscous effects and negligible inertial ones (referred as torsional Couette flows). Due to the small axial clearances between the disks, this type of flows has been considered only recently numerically by Andersson and Lygren [4] using LES, Haddadi and Poncet [5] using RANS modeling and experimentally by Pellé and Harmand [3]. More attention has been turned to the regimes with unmerged boundary layers. Batchelor [6] showed that the flow structure may be divided into thee regions: two unmerged bound-

$\overline{1 \text { poncet} @ 13 m . u n i v-m r s . f r, ~ T e l .33 ~(4) ~ 91.11 .85 .55, ~ F a x .33 ~(4) ~ 91.11 .85 .02 ~}$ 
ary layers with a rotating core of fluid in which radial and axial velocities are negligible. The core of fluid is characterized by the core-swirl ratio (or entrainment coefficient), denoted $\beta$ (or $K$ ), which is the ratio between the tangential velocity of the core $V_{\theta}$ and the tangential velocity of the disk at the same radius $\omega r$. Poncet et $a l$. [7] shows experimentally and numerically that $\beta_{0}=\beta(Q=0)=0.438$ ( $Q$ the voluminal flowrate) whatever the radial position and the interdisk spacing for turbulent flows with unmerged boundary layers without throughflow. However, Stewartson [8] found a different solution with two unmerged boundary layers and a tangential velocity equal to zero everywhere excepted on the rotor side. Measurements by Brady and Durlofsky [9] showed that a shrouded cavity facilitates a Batchelor type flow while unshrouded air-gap gives more favorably a Stewartson type flow.

About the convective heat transfer, adding a stator results generally in a decrease of the local convective heat transfers for several reasons explained in the very detailed synthesis of Owen and Rogers [10]. In a Couette type flow, the centrifugal and centripetal boundary layers are merged, resulting in a strong viscous flow with low velocities compared to the free disk for the same rotational speed. For turbulent Couette flows (regime III after Daily and Nece [2]), Owen and Rogers [10] proposed for the averaged Nusselt number over the rotor:

$$
N u_{a v_{I I}}=\frac{0.0308}{\pi} G^{-1 / 4} R e^{3 / 4}
$$

When there is a Batchelor type flow in the air-gap $\left(G>0.2112 R e^{-3 / 16}\right)$, the rotating core of fluid gives a decrease in the shear stresses at the rotating walls and the heat transfers decrease again. For turbulent Batchelor flows (regime IV), Daily and Nece [2] proposed for the averaged Nusselt number over the rotor:

$$
N u_{a v_{I V}}=\frac{0.0545}{\pi}\left(\frac{G}{2}\right)^{0.1} R e^{4 / 5}
$$

For an increasing air-gap, a Stewartson type flow occurs and the rotating core tends to disappear, which gives an increase in the local heat transfer. They reach results obtained for a free disk when the stator is sufficiently far away from the rotor not to alter the flow. Pellé and Harmand [3] performed extensive measurements of the heat transfer coefficients along the rotating disk in a rotor-stator system with an open end air-gap. Their results showed that none of the previous correlations of Owen and Rogers [10] or Daily and Nece [2] for all four flow regimes provide fully satisfactory results for the whole disk surface in such configuration.

When an axial flow is superimposed on the base rotor-stator flow, the same four flow regimes can be observed but with different boundaries. A radial outflow can cause a premature transition to turbulence $[11,10]$. Daily et al. 
[12] measured the averaged velocity profiles in the case of an outward flow in an unshrouded system with a radial outlet. They put forward the importance of the outflow on the development of the rotor boundary layer and on the coreswirl ratio. They showed that the geometry of the hub at the inlet modifies the mean velocity components but not the pressure distribution within the cavity. They proposed also the following correlation for $R e=6.9 \times 10^{5}, G=0.052$ and 0.069 and various flowrates:

$$
\frac{\beta}{\beta_{0}}=\frac{1}{1+12.74 \lambda_{t}\left(\frac{R_{e x t}}{r}\right)^{13 / 5}}
$$

with $r$ the local radius and $\lambda_{t}=C_{w} R e^{-0.8}$ the turbulent flow parameter, $C_{w}=Q /\left(\nu R_{e x t}\right)$ being a nondimensional flowrate. More recently, Poncet et al. $[7,13]$ have investigated experimentally and numerically the influence of a superimposed throughflow which can be either centrifugal or centripetal in the case of a shrouded cavity with axial inlet and outlet. In particular, they proved that centrifugal throughflow can make the flow changes from Batchelor to Stewartson type flow for sufficiently large flow rates. The flow becomes as Stewartson described it when the local flowrate coefficient, defined by $C q_{r}=$ $\frac{Q R e_{r}^{1 / 5}}{2 \pi r^{3} \omega}$, is higher than $0.025\left(R e_{r}=\omega r^{2} / \nu\right.$ the local Reynolds number $)$. This result does not depend on $G$ and is valid as long as the flow remains turbulent with unmerged boundary layers. If the flow is a Batchelor type flow, Poncet et al. [7] have proposed the following equation for $\beta$ :

$$
\beta=2 \times\left(0.63-5.9 \times C q_{r}\right)^{5 / 7}-1
$$

For a Stewartson-type flow, Poncet et al. [13] found that $\beta$ decreases exponentially with increasing values of $C q_{r}$ following the equation:

$$
\beta=0.032+0.32 \times e^{\left(-C q_{r} / 0.028\right)}
$$

The rotating core flow is suppressed for $C_{w}=0.219 R e^{0.8}\left(r / R_{e x t}\right)^{2.6}[14]$. This value for the nondimensional flowrate corresponds to the entrainment flow for a Von Kármán flow over a free disk. For some sets of parameters, both Batchelor and Stewartson may be observed within the gap. Owen and Rogers [10] observed that for radii larger than $\left(\lambda_{t} / 0.219\right)^{5 / 13}$, the inviscid core becomes negligible. The latter conclusion have recently revised by Da Soghe and co-workers [15]. The authors pointed out, by means of a validated CFD campaign, that the transition from the Stewardson to the Batchelor regime take place when $\left(\lambda_{t}\right)^{-13 / 5}<0.32$.

The main effect of an unshrouded cavity on the hydrodynamic field is that the flow pumped from the rotor leaves the cavity to the external surroundings and ingress may then occur to supply the entrainment demands of the rotat- 
ing flow. At the periphery of the cavity, there can therefore be both inflow along the stator and outflow on the rotor. The superposed flow necessary to prevent ingestion is strongly dependent on the shroud clearance $j$ following the empirical relationship:

$$
C_{w}=0.61 \frac{j}{R_{e x t}} R e
$$

Owen et al. [16] proposed the limiting aspect ratio beyond which the moment coefficient on the rotor is unaffected by the presence of the stator for an unshrouded cavity:

$$
G=1.05 R e^{-0.2}
$$

with $R e$ up to 4.1 millions, $G$ in the range [0.005-0.604] and nozzle diameter to interdisk spacing ratio between 2.25 and 51 .

In the literature, a huge amount of heat transfer data is available but it is often restricted to cooling of a stationary disk by jet impingement. One can cite, among others, the work of Gao and Ewing [17], who have experimentally studied the effect of confinement on the heat transfer due to an impinging jet. It corresponds to an interdisk cavity without any rotation, with $0.025 \leq e / D \leq 6$ and $17000 \leq R e_{j} \leq 28000\left(R e_{j}=V D / \nu\right.$ the jet Reynolds number with $V$ the jet velocity and $D$ the jet diameter). The presence of the confining plate had no significant effect on the heat transfer when $e / D \geq 1$. However, for $e / D \leq 0.5$, the heat transfer was reduced up to $50 \%$. That decrease is caused by a change in the turbulent fluctuations near the heat transfer surface. Only few groups of researchers have concentrated their efforts to investigate the problem of a jet impinging onto a rotating disk with confinement effects. Sara et al. [18] have experimentally investigated the mass transfer between an impinging jet and a rotating disk in a confined system by naphtalene sublimation. Their parameters are such that: $2 \leq e / D \leq 8,17000 \leq R e_{j} \leq 53000$ and $34000 \leq R e \leq 120000$. In those ranges of parameters, authors conclude that the mass/heat transfers increase by increasing $R e$ and $R e_{j}$ even if with their low rotational Reynolds numbers, the jet is dominant. One can cite also Minagawa and Obi [19], who have been interested in the turbulence development in this configuration. In fact, in all available studies about convective heat transfer in configuration where effects of a rotating disk and jet impingement are coupled, authors [20,21] have distinguished three regions:

- Region 1: the area of the disk where the jet influence on the heat transfer is the greatest near the impingement point;

- Region 2: a mixture between regions 1 and 3;

- Region 3: the area where rotation has the greatest effect far from the impingement point at high radii. 
Chen et al. [21] have also concluded that the location of those three zones depends on the ratio between the jet and the rotation mass flow rates. They have also noted that the heat transfer on the rotating disk is modified by the jet for rotational Reynolds numbers lower than $2 \times 10^{5}$. For greater values of $R e$, the jet does not affect the local heat transfer distribution. More recently, Pellé and Harmand [22] measured the local convective heat transfer coefficient along the rotor by infrared thermography. They considered an impinging jet in an unshrouded rotor-stator cavity for $R e_{j}=4.16 \times 10^{4}, R e=\left[2 \times 10^{4}-5.16 \times 10^{5}\right]$ and $G=[0.01-0.16]$. Whatever the flow parameters, the jet always enhances the heat transfer compared to the no-jet configuration. They found that the size of region 1 near the stagnation point strongly depends on $G$, while at outer radii, the local Nusselt number on the rotor depends on both $R e_{j}$ and $R e$. They proposed correlations for the averaged Nusselt numbers for different ranges of aspect ratios showing that it is directly linked to the transition between a purely centrifugal flow for $G \leq 0.02$, to a Batchelor type flow $(0.04 \leq G \leq 0.08)$ and then to a Stewartson type flow for $G=0.16$.

As far as unshrouded rotor-stator systems with impinging jet are concerned, there is a lack of information in the literature, mainly for the hydrodynamic field, as pointed out by the recent review of Harmand et al. [23]. Numerical simulations are complex due to the superimposition of complex elementary phenomena (impinging jet, high rotation rates, confinement effects, unshrouded cavity ...). Experimental works are also usually done for shrouded cavities. As Brady and Durlofsky [9] have shown, the presence of a shroud can be of great effect on the flow structure and so on the heat transfers. In this work, one has both experimentally and numerically investigated the flow structure and the heat transfer in an unshrouded rotor-stator system for a wide range of jet velocities and rotational speeds at a low air-gap thickness, the one which gives the lowest heat transfer when there is no impinging jet [3]. Lot of informations about rotating systems with or without jet can be found in the reviews by Owen and Rogers [10] or more recently by Launder et al. [24] and Childs [14] for fluid flow aspects and Shevchuk [25] for heat transfer processes. The reader can refer also to the review of Harmand et al. [23] on the fluid flow and convective heat transfer within rotating disk cavities with impinging jet.

The paper is organized as follows: the experimental procedure and the numerical modeling are briefly described in Sections 2 and 3 respectively. The results about fluid flow aspects are presented and discussed in Section 4 in terms of flow structures, mean and turbulent flow fields. Comparisons about the heat transfer distribution are provided in Section 5. Finally some conclusions and closing remarks are provided in Section 6. 


\section{Experimental procedure}

\subsection{Experimental setup}

The experimental set-up in this study was the same than previously detailed in $[3,22]$ and shown in Figure 1 . The rotor has a $R_{e x t}=310 \mathrm{~mm}$ radius and its rotational velocity could be changed with a frequency variator. Rotor is made with aluminum, chosen for its high thermal conductivity and its low density. A $p=2.5 \mathrm{~mm}$ layer of zircon whose thermal conductivity is $\lambda_{z i r}=0.7 \mathrm{~W} / \mathrm{mK}$ was deposited on the cooled side of the rotor by plasma projection. Four infrared emitters were placed on the bottom of the disk in order to heat it until cooled surface reached about $80^{\circ} \mathrm{C}$. A stationary disk faced the rotor at a distance $e=6.2 \mathrm{~mm}$ for which the dimensionless spacing interval is $G=e / R_{e x t}=0.02$. It guarantees that the stator clearly affects the fluid flow within the cavity, as $G$ remains far below the limiting value $G=0.0752$ obtained after Equation (7) for the maximum Reynolds number considered here (Table 1). The stationary disk had the same diameter as the rotating disk and a central opening $(D=26 \mathrm{~mm})$ was pierced at the stator to allow passage of a long pipe linked to a centrifugal blower, which was used to impose an axial flow. The jet aspect ratio was then $e / D=0.25$. The jet mass flow rate was controlled by pressure losses measurements, which were previously correlated to the mass flow rate, and adjusted by creating a pressure loss at the blower suction.

\subsection{Flow control parameters}

Apart from the two geometrical parameters $e / D$ and $G$, the base flow depends also on the jet and rotational Reynolds numbers defined respectively by:

$$
R e_{j}=\frac{V D}{\nu} \quad R e=\frac{\omega R_{e x t}^{2}}{\nu}
$$

with $\nu$ the fluid kinematic viscosity and $V$ the jet axial flow velocity imposed at the inlet. For numerical investigations, it is also convenient to use a nondimensional flowrate $C_{w}=\frac{Q}{\nu R_{e x t}}$ ( $Q$ the voluminal flowrate) instead of $R e_{j}$ and which can be combined with Re to form the turbulent flow parameter $\lambda_{t}$. To enable direct comparisons between the axial flow due to the impinging jet and the tangential flow due to the rotation of the disk, one define also the rotation parameter $N$. 


$$
\lambda_{t}=C_{w} R e^{-0.8} \quad N=\frac{\omega R_{e x t}}{V}
$$

All the values considered for these parameters are summed up in Table 1.

\subsection{Measurement technique for the hydrodynamic field}

Fluid velocity was measured by PIV measurements. Complete descriptions of the PIV experimental setup and measurements are given in Nguyen et al. [26], such that only a brief review is described here. The PIV system consisted of a double-pulsed Nd:YAG laser, a CCD camera mounted with a Nikkor lens of $105 \mathrm{~mm}$ and $f / 5.6$ aperture, and a synchronizer. The laser sheet thickness was about $1 \mathrm{~mm}$ created by a cylindrical and a spherical lens. This thickness was optimized to generate particle intensity and reduce the loss of particle image pairs. An olive oil droplet generator (TSI 9307) generated particles with a mean diameter of $1 \mu \mathrm{m}$ to seed the inlet of the centrifugal blower. PIV images were captured by the TSI PowerView Plus $4 \mathrm{MP}$ camera with a resolution of $2048 \times 2048$ pixels and a pixel size of $7.4 \times 7.4 \mu \mathrm{m}^{2}$. The time interval between the first and second exposures was chosen from 15 to $150 \mu \mathrm{s}$, yielding maximum particle displacements of 6 pixels.

PIV measurements were performed at three $(r, \theta)$ planes defined $z_{1} / e=0.23$ (near the rotor), $z_{2} / e=0.53$ (the middle plane) and $z_{3} / e=0.84$ (near the stator) as shown on Figure 2. At each axial plane, PIV images were separately captured at three different regions, i.e. regions 1,2 and 3, with similar flow conditions. The PIV measurements of regions 1,2 and 3 covered the flow regions in the $(x, y)$ plane with $x / R_{\text {ext }}$ ranging from -0.37 to $-0.04,-0.71$ to -0.38 and -1 to -0.72 , respectively. For the values of $R e$ and $R e_{j}$ considered here, 500 image pairs were recorded for each run at a sampling rate of $1 \mathrm{~Hz}$ to ensure that the velocity fields obtained from the image pairs are statistically independent.

Image acquisition and processing were performed with TSI Insight TM 3G software (version 9.1). The PIV images were analysed by a recursive Nyquist rectangular grid algorithm with two iterations and $50 \%$ window overlap. The first-pass and the second-pass interrogation windows were square interrogation spots of $64 \times 64$ pixels and $32 \times 32$ pixels, respectively. The final number of velocity vectors was $110 \times 110$ vectors. In the velocity computation, $2 \mathrm{D}$ displacement fields were computed from the correlation map with a Gaussian peak fit [27] for sub-pixel accuracy and were validated by the signal-to-noise ratio of 1.5. Statistical validation tools using a median filter [28] and a standard deviation filter were performed between the iterations to remove erroneous vectors, and then fill in the blanks by interpolation.

The percentage of bad vectors calculated as the average over the number of PIV vector fields was about $2 \%$. The uncertainty in the PIV measurements, 
calculated using the error analysis developed by Coleman and Steele [29], were estimated to be less than $9 \%$ of the mean jet velocity in region 1 and typically less than $4 \%$ and $6 \%$ in regions 2 and 3 , respectively.

\subsection{Measurement technique for the temperature field}

\subsubsection{Temperature measurements}

An AGEMA 900 infrared camera is situated one meter above the rotor (surface emissivity $\varepsilon_{r}=0.93 \pm 0.01$ ) and recorded the rotor surface temperatures with a frequency of $35 \mathrm{~Hz}$.

Two T-type thermocouples, located at two different radii $r=0$ and $r=0.3$ $\mathrm{m}$, were used to measure temperatures at the bottom of the disk at the aluminium/zircon interface. Measurements were realized using a Graphtec GL 200 acquisition system whose uncertainty on the temperature is $\pm 0.5 \mathrm{~K}$ for T-type thermocouples. The thickness of the aluminium layer is such that the temperature at the zircon/aluminum interface is homogeneous for our operating conditions. Thermocouples give a difference temperature less than $1 K$. The reference air temperature $T_{\infty}$ was measured by a K-type thermocouple placed outside the test-rig, far enough from the heating system but near from the backside of the stator, in order to measure the temperature of the air which will enter in the air-gap. That temperature is assumed constant during a test, due to the placement of the experimental apparatus in a very large room. The absolute error for the air temperature is estimated at $\pm 1 \mathrm{~K}$ for the used acquisition system.

Two special T-type thermocouples located at the bottom of the stator to put them in constant contact with the stator surface inside the air-gap were directly linked to the acquisition system. They were placed at radii of $0.05 \mathrm{~m}$ and $0.3 \mathrm{~m}$. As for the measurements at the zircon/aluminium interface, the absolute error for the stator temperature $T_{\text {stator }}$ is $\pm 0.5 \mathrm{~K}$ according to the data acquisition system's specifications. Due to the high conductivity material which is used for the stator, temperature difference between the thermocouples is under $1 \mathrm{~K}$.

\subsubsection{Heat transfer coefficient}

The wall heat flux on the rotor surface can be obtained by solving the heat equation in the zircon layer using a finite-difference method. The boundary conditions applied are the surface temperatures $T_{s}$ recorded by an infrared camera (watching the rotor through a fluorspar window placed inside the stator) and the interface (aluminum/zircon) temperatures recorded by thermocouples $T(z=p)$. The convective heat flux can be deduced by solving a 
thermal balance equation for each point on the disk surface and the local heat transfer coefficient is then obtained. The choice of the reference temperature for the Nusselt number calculation is made taking into account several reasons. The best choice in such a discoidal system [10] is the adiabatic wall temperature $T_{a d}$, which can be either measured or calculated by:

$$
T_{a d}=T_{0}+\frac{\operatorname{Pr}^{1 / 3}(\omega r)^{2}}{2 C_{p}}
$$

where $T_{0}$ is the temperature of air which comes to feed the boundary layer near the rotor and $\operatorname{Pr}=\nu / \alpha$ the Prandtl number ( $\alpha$ the thermal diffusivity of the fluid). Measuring that adiabatic temperature inside the air-gap requires the insertion of a thermocouple, which can modify the flow and so the heat transfers. Moreover, as our system is not closed and air comes into the air-gap from the test room by the jet, $T_{\infty}$ can be chosen for $T_{0}$, the ambient temperature of the test room. Moreover, an estimation of the maximum reached by $\frac{\operatorname{Pr}^{1 / 3}(\omega r)^{2}}{2 C_{p}}\left(C_{p}\right.$ the mass thermal capacity) shows that the temperature increase due to friction effects is under $0.3 \mathrm{~K}$. So it is assumed that friction effects are negligible for our operating conditions and $T_{\infty}$ can be taken as the reference temperature.

The local Nusselt number can then be expressed as:

$$
\begin{aligned}
& N u=\frac{\varphi_{c d}-\varphi_{\text {rad }}}{T(r)-T_{\infty}} \times \frac{D}{\lambda_{\text {air }}}=\frac{h D}{\lambda_{\text {air }}} \\
& \varphi_{c d}=\lambda_{z i r}\left[\frac{\partial T(r)}{\partial z}\right]_{z=0} \\
& \varphi_{\text {rad }}=\sigma \frac{F \varepsilon_{r} \varepsilon_{s}}{1-F^{2}\left(1-\varepsilon_{r}\right)\left(1-\varepsilon_{s}\right)}\left[T(r)^{4}-T_{\text {stator }}^{4}\right]
\end{aligned}
$$

where $\varphi_{c d}$ and $\varphi_{\text {rad }}$ are the conductive and radiative heat fluxes respectively, $h$ is the convective heat transfer coefficient, $\lambda_{\text {air }}$ and $\lambda_{z i r}$ the thermal conductivities of air and zircon, $\varepsilon_{r}$ and $\varepsilon_{s}$ the rotor and stator emissivities, $\sigma$ the Stefan-Boltzman constant and $F$ the view factor.

The reader can refer to the previous paper of Pellé and Harmand [3,22] for more details about the temperature measurement set-up. Taking into account all uncertainties, Pellé and Harmand [30] showed that the Nusselt numbers can be determined with an uncertainty under $30 \%$.

To insure that the experimental set-up would produce reliable results, a preliminary study on a single rotating disk configuration without impinging jet was conducted. The temperature distribution along the rotor side is isothermal. The results obtained are close to those of Dorfman [31] in this very well-known configuration as shown on Figure 3 for $R e=5.16 \times 10^{5}$. 


\section{$3 \quad$ Statistical modeling}

Despite the relative simplicity of the geometry, rotating disk flows contain a complex physics, which makes their modeling a very challenging task for numerical methods. The high rotation rates reached in real rotating machineries induce the coexistence of laminar, transitional and turbulent flow regions, very thin boundary layers along the walls whose thickness scales like $\sqrt{\nu / \Omega} \ldots$ An impinging jet yields the flow even more complex because of the interaction between the jet and the secondary rotor-stator base flow. The presence of a confinement stationary disk may also induce large recirculation and flow ingress, which are also severe conditions for turbulence modeling methods. That is why one use in the present work the third version "RSM3" of the RANS model developed by Elena \& Schiestel [1], which has proven its superiority in many rotating flow arrangements $[1,32,7,33,34,13]$. Its predictions will be compared to those of the $k-\omega$ SST model available within CFX.

\subsection{The differential Reynolds Stress Model (RSM)}

\subsubsection{Description of the RSM model}

Our approach is based on one-point statistical modeling using a low Reynolds number second-order full stress transport closure derived from the Launder and Tselepidakis [35] model and sensitized to rotation effects by Elena \& Schiestel [1]. This approach allows for a detailed description of near-wall turbulence and is free from any eddy viscosity hypothesis. The general equation for the Reynolds stress tensor $R_{i j}$ can be written:

$$
\frac{d R_{i j}}{d t}=P_{i j}+D_{i j}+\Phi_{i j}-\epsilon_{i j}+S_{i j}
$$

where $P_{i j}, D_{i j}, \Phi_{i j}$ and $\epsilon_{i j}$ respectively denote the production, diffusion, pressurestrain correlation and dissipation terms. The term $S_{i j}$ takes into account the implicit effects of rotation on turbulence. The seventh equation to be solved is the one for the dissipation rate equation $\varepsilon$ under the form proposed by Launder and Tselepidakis [35].

The extra term $S_{i j}$ accounts for implicit effects of rotation on turbulence, which are directly linked with one-point correlation dynamics. It is decomposed into four terms:

$$
S_{i j}=\Phi_{i j}^{(R)}+D_{i j}^{R}+B_{i j}+J_{i j}
$$


The term $\Phi^{(R)}$ is a linear part of the pressure-strain correlation term sensitized to the dimensionality tensor, which represents the spatial properties of the turbulent structures. $D^{R}$ is an inhomogeneous diffusion term, which slows down the tendency to bidimensionalization for wall bounded flows. $B$ is a homogeneous source term, which rectifies the pressure-strain correlation and which acts only in case of strong rotation. It produces spectral phase scrambling (angular dispersion). The rotation also reduces the energy transfer from large to small turbulent scales. It is modeled through an inverse flux $J$ considered as isotropic for high Reynolds numbers. These terms allowed some improvements of results in an enclosed rotor-stator cavity [1]. The influence of the terms $\epsilon, P, D, D^{R}$ and $J$ on the turbulence kinetic energy budgets has been quantified and discussed in details for enclosed rotor-stator disk flows in [32]. The entire RSM3 model is described in in [1,32].

For the thermal field, it is supposed that density is not significantly affected by temperature differences. Temperature is then considered as a passive scalar. Forced convection is indeed the main mechanism for the heat transfer in the present system. A similar approach has been used with success in a similar rotor-stator cavity by Poncet and Schiestel [34] and in Taylor-CouettePoiseuille flows by Poncet et al. [33]. The following equation for temperature is then solved:

$$
\frac{\partial T}{\partial t}+V_{j} T_{, j}=\alpha T_{, j j}-F_{j, j}^{t}
$$

where $F_{i}^{t}$ is the turbulent heat flux approximated by a gradient hypothesis with tensorial diffusive coefficient:

$$
F_{i}^{t}=-c_{t} \frac{k}{\varepsilon} R_{i j} T_{, j}
$$

where $c_{t}=c_{\mu} / P r_{t}=0.1$ with $c_{\mu}=\nu_{t} \varepsilon / k^{2}=0.09$ a coefficient used to define the turbulent viscosity $\nu_{t}$ and $P r_{t}$ the turbulent Prandtl number assumed to be constant at 0.9. The effects of the anisotropy of the turbulence field and the effects of rotation are already included in $k R_{i j} / \varepsilon$ for most of them.

\subsubsection{Numerical method}

The numerical solver employed here is based on a finite volume method originating from the TEAM (Turbulent Elliptic Axisymmetric Manchester) code and modified later by Elena and Schiestel [1] for rotating flows. The convection and diffusion terms are discretized with the power-law scheme and the velocity-pressure linkage is solved using the SIMPLER algorithm. The computer code is steady elliptic and the numerical solution proceeds iteratively. The code is used in its steady axisymmetric version (the unsteadiness of the 
flow has first been checked but the final results remained steady). To overcome possible stability problems due to high rotation rates, several stabilizing techniques were introduced by Elena and Schiestel [1] in the numerical procedure. Thus, the three mean velocity components are discretized on a staggered grid, whereas the six Reynolds stress tensor components are collocated at the pressure node allowing block solution, which strongly enhances stability. The stress component equation system is solved using matrix block tridiagonal solution, which is necessary to reach the convergence. The Reynolds stress tensor is decomposed appropriately to enable a diffusive formulation reported into the momentum equations. Finally, regular and inertial relaxation parameters are also introduced with different relaxation coefficients for each variable. The calculation is initialized using realistic data fields, which satisfy the boundary conditions. About 50000 iterations (6.5 seconds per iteration on the M2P2 cluster with 2 Xeon quadcore at $3 \mathrm{GHz}$ ) are necessary to obtain the numerical convergence of the calculation. A $200 \times 80$ structured mesh in the $(r, z)$ frame proved to be sufficient for all cases to get grid-independent solutions $[7,32,34]$. The mesh is built according to geometrical series variations allowing a well-controlled refinement near the walls. The pressure and the tangential velocity component are solved on the same mesh points, while two different staggered grids are used for the two other velocity components. The reader can refer to the PhD thesis of Poncet [32] for more details. For the highest values of the Reynolds numbers $\left(R e=5.32 \times 10^{5}, R e_{j}=4.3 \times 10^{4}\right)$, it provides wall coordinates $z^{+}$along the rotor and the stator lower than 0.5 and 0.8 respectively, which ensures a correct description of the viscous sublayers. More details about the numerical method are given in [1,32].

\subsubsection{Boundary conditions}

The boundary conditions are as follows:

- Along the symmetry axis:

$$
\begin{array}{r}
\frac{\partial \phi}{\partial r}=0, \quad \phi=V_{r}, V_{\theta}, V_{z}, k, \varepsilon, R_{r r}, R_{\theta \theta}, R_{z z} \\
R_{r \theta}=R_{r z}=R_{\theta z}=0
\end{array}
$$

- At the walls: $V_{r}=V_{z}=k=R_{i j}=0$ for $(i, j)=(r, \theta, z)$ and $\varepsilon=2 \nu\left(\sqrt{k}_{, i}\right)^{2}$. $V_{\theta}=0$ on the stationary disk and $V_{\theta}=\omega r$ on the rotor.

- At the inlet: $V_{\theta}$ is supposed to vary linearly from zero on the stationary wall up to $\omega r$ on the rotating wall. When a throughflow is enforced, a turbulent Poiseuille profile is then imposed for the axial velocity $V_{z}$ without any preswirl. A given level of turbulence intensity is also imposed corresponding to a turbulence kinetic energy at the inlet equal to $0.01(\omega r)^{2}$ and a turbulence Reynolds number equal to $R e_{t}=k^{2} /(\nu \varepsilon)=1500$.

- At the outlet: the pressure is fixed, whereas the derivatives for all the other 
independent quantities are set to zero if the fluid leaves the cavity, and fixed external values are imposed if the fluid re-enters the cavity. The continuity equation is then used to determine the outward velocity component. In this case, the boundary condition is of mixed type and a special technique is used to enhance stability [32].

The flow in the similarity area is practically not sensitive to the shape of velocity profiles or to the intensity level imposed at the inlet [32]. The turbulence levels calculated inside the cavity are indeed always larger than those imposed at the inlet. Moreover, these choices are justified by the wish to have a model as universal as possible.

For the thermal field, the air flow $(\operatorname{Pr}=0.7)$ at the inlet enters the cavity at $T_{\infty}=20^{\circ} \mathrm{C}$, which is also the temperature imposed on the stator surface. The temperature of the rotor is fixed to $T_{\text {rotor }}=80^{\circ} \mathrm{C}$. At the periphery of the cavity, if ingress occurs, the fluid reenters the cavity at $T_{\infty}$.

\subsubsection{Validation of the RSM model}

This version of the RSM has been already fully validated in various rotating flow arrangements: von Kármán flows between counter-rotating stirrers, Taylor-Couette-Poiseuille flows between concentric cylinders, rotor-stator flows with axial inward or outward throughflow $[7,13]$, with merged or unmerged boundary layers and with heat transfer [34]. Nevertheless, to show the capability to predict such complex flows, the predictions of the present RSM have been compared to a low-Reynolds number $k-\epsilon$ model and to the LDV measurements of Poncet [32]. A shrouded cavity of aspect ratio $G=0.036$ is considered with a given rotational Reynolds number $R e=1.04 \times 10^{6}$ and 4 values of the flowrate coefficient. Axial profiles of the mean radial $V_{r} /(\omega r)$ and tangential $V_{\theta} /(\omega r)$ velocity components and the corresponding Reynolds stress tensor components are shown in Figure 4 at $r / R_{e x t}=0.56$. The Reynolds stress tensor components are defined by: $R_{r r}^{*}=\overline{v_{r}^{\prime 2}} /(\omega r)^{2}, R_{\theta \theta}^{*}=\overline{v_{\theta}^{\prime 2}} /(\omega r)^{2}$, $R_{r \theta}^{*}=\overline{v_{r}^{\prime} v_{\theta}^{\prime}} /(\omega r)^{2}$.

The flow dynamics will not be discussed in details in this section. It is noteworthy that the RSM predicts quite well the mean and turbulent fields by catching the main characteristics of turbulent rotor-stator flows:

- for $C_{w}=0$ (Fig.4a), the cavity is completely closed. A Batchelor flow structure is obtained with two boundary layers separated by an inviscid rotating core. The Ekman layer on the rotor is centrifugal and by conservation of mass, the Bödewadt layer along the stator is centripetal. The boundary layer thicknesses as well as the extrema of $V_{r}$ are well predicted by the RSM. Turbulence is mainly concentrated along the disks. The extrema of the turbulent intensities are also well predicted even for the cross component, which is particularly difficult to catch by classical two-equation models 
(see in Da Soghe et al. [36]).

- for $C_{w}>0$ (Fig.4b to d), an axial outward flow is superimposed on the base rotor-stator flow. When one increases $C_{w}$, the flow structure switches progressively to a Stewartson type flow $\left(C_{w}=5929\right.$, Fig.4c) with only one boundary layer on the rotor and a quasi zero tangential velocity outside. The main flow is centrifugal due to the impinging jet. The highest turbulent levels are observed along the rotating disk and vanish towards the stator. For these sets of parameters, the RSM predicts quite well the mean and turbulent fields.

For all cases, the $k-\epsilon$ model, which is blind to any rotation effects, suffers from serious deficiencies with an overestimation of the mean tangential velocity when an outward flow is imposed. Moreover, it strongly overestimates the turbulent intensities within the air-gap for all the test cases. The RSM can now be used with confidence for the impinging jet flow problem.

\subsection{The $k-\omega$ SST model available within CFX}

CFD steady state calculations have been performed with the commercial 3D Navier-Stokes solver Ansys CFX v.14.5. Compressibility effects have been taken into account and a second-order upwind advection scheme has been used. The fluid (air) has been modeled as ideal gas and the properties of specific heat capacity, thermal conductivity and viscosity have been assumed as constants. The energy equation has been solved in terms of total temperature and viscous heating effects have been accounted for. The $k-\omega$ SST turbulence model is used, in its formulation made available by the CFD solver, with an automatic near wall treatment which recovers fully low Reynolds approach as the dimensionless first cell wall distance is below 1 everywhere.

Calculations have also been performed with a modified version of its model including a curvature correction term. Recently, Smirnov and Menter [37] have indeed applied to the $k-\omega$ SST the modification proposed by Spalart and Shur [38], to sensitize the two-equation turbulence model to the rotation and curvature effects. Basically, the model correction results in a multiplicative factor of the transport equation production term. This factor is expressed as a complex function of the stress tensor $S$ and the vorticity tensor $\Omega_{\text {vort }}$. The results will not be shown in the following as, for Test cases 4,5 and 6 , it provides results undistinguishable from those obtained with the classical $k-\omega$ SST.

The convergence of solutions has been assessed by monitoring domain mass imbalance (below 0.001\%) and residuals (below $10^{-7}$ ). Furthermore the runs have been stopped when the pressure level and other physical quantities on different locations, reached a steady state. The grid consists in a structured mesh $(200 \times 80$ in the $(r, z)$ frame). As the flow is statistically homogeneous 
in the tangential direction, the numerical domain is limited to a $20^{\circ}$ sector and the mesh counts 20 computational nodes in the tangential direction. A cyclic condition has been imposed at the domain periodic planes while no-slip conditions have been applied on solid walls. The pressure boundary condition has been imposed at the outlet while mass flow rates were fixed at the inlet. Fixed temperature level $\left(80^{\circ} \mathrm{C}\right)$ has been prescribed at the rotor disk surface. About 6000 iterations (72 seconds per iteration on 4 cores of a Intel Xeon E5645 2.4 GHz) are required to reach the convergence.

\section{Hydrodynamic flow field}

The experimental results are discussed in terms of the hydrodynamic field and compared to the predictions of the RSM and $k-\omega$ SST models for some characteristic test cases among the nine defined in Table 1.

\subsection{Flow structure}

Figure 5 presents the mean streamline patterns obtained by the RSM model in the meridian $(r, z)$ plane for all test cases (Table 1$)$. The air jet squirtes from the center of the stator and impinges the rotor. After the impingement, the fluid is deflected and flows radially along the rotor. This radial outflow, which is enhanced due to the combination of the jet flow and centrifugal effects, is confined by a large recirculation zone appearing along the stationary disk. Poncet [32] showed that the size of this recirculation bubble depends mainly on the aspect ratio $G$ of the cavity, which could explain why it is almost constant in the present study. Whatever the flow parameters, this jet-dominated area (Region 1) observed close to the rotation axis extends to a dimensionless radius $r / R_{e x t} \simeq 0.14$. For larger radii, the flow is purely centrifugal with streamlines parallel to the disks. In that case, this flow structure is sometimes called a Stewartson flow structure by abuse of language $[32,7,13]$. Its denomination is used to mention that looking at the tangential velocity profile, there is only one boundary layer on the rotating disk. For some particular test cases $(4,5,7,8,9)$, a recirculation zone is observed on the stator side. Equation (6) predicts that the minimum values for the flowrate coefficient $C_{w}$ preventing the formation of such recirculation, are respectively $C_{w}=403, C_{w}=3245$ and $C_{w}=6490$ for Test cases $1-3,4-6$ and $7-9$. The only discrepancy here is obtained for Test case 6 , for which the flowrate $C_{w}=2833$ is slightly weaker than the predicted value $C_{w}=3245$. Thus, in these particular cases, some fluid does not leave the cavity at its periphery and flows radially inward along the stationary disk by conservation of mass. The flow exhibits then a Batchelor-like structure with two boundary layers, one on each disk. It is clear 
from the streamline patterns that the radial extent of this recirculation zone at the periphery decreases when the rotation parameter $N$ decreases. For Test case 7 ( $N=2.597$, Fig.5g), the flow is dominated by rotation effects for $r / R_{\text {ext }}$ larger than 0.51, whereas for Test case $5(N=0.866$, Fig.5e), the fluid flows inwards on the stator only up to $r / R_{e x t}=0.89$.

Figure 6 shows the streamline patterns for Test cases 4 to 6 obtained by the $k-\omega$ SST model. Qualitatively, the same results are obtained by this model. Nevertheless, one can notice that the recirculation along the stator at low radii after the jet impingement is much larger than the one observed by the RSM. Its size is besides not so sensitive to the value of the jet Reynolds number $R e_{j}$. This overprediction of the size of the recirculation bubble has already pointed out by Da Soghe et al. [36]. Secondly, some backflow was observed along the stator by the RSM for a given set of parameters. It is also the case here for Test case 4 but this backflow does not penetrate so much the cavity.

Figure 7 shows the measured time-averaged velocity vectors (normalized by $V)$, in different $(x, y)$ planes near the rotor and near the stator for two characteristic Test cases 1 and 7. Figure 7a,b are relative to a low rotational Reynolds number while Fig. $7 \mathrm{c}, \mathrm{d}$ are for the highest $R e$ in our tested range. Images on the left handside are for the plane near the rotor and images on the right hand side are for the plane near the stator. For a low rotational speed, it is clearly shown on Fig. 7a,b that the flow is mainly centrifugal everywhere inside the air-gap, except near the impingement point $(x=y=0)$ where a recirculation region is visible. The main difference with the Fig. 7c,d is the appearance of rotational effects for approximately $x / R_{e x t} \geq 0.5$ whatever the observation plane. However, near the stator, velocities seems to be essentially tangential for those radius while a centrifugal component is again visible near the rotor. The same recirculation region near the jet impingement region is present and seems not to depend on the rotational speed. A more precise analysis is proposed thereafter by extracting mean velocity profiles along the radius.

\subsection{Mean flow field}

One interesting mean quantity for engineers is the radial distribution of the core-swirl ratio $\beta$ known also in the literature as the entrainment coefficient sometimes denoted $K$. It is defined as the ratio of the time-averaged tangential velocity at mid-gap $(z / e=0.5)$ and the local tangential velocity of the rotor $\omega r$ at the same radius. For flows with unmerged boundary layers (Batchelor flows), $\beta$ can be indeed directly linked to the radial pressure gradient within the cavity and thus to the axial thrusts applied on the rotor [7], which can be useful in real turbines. Figure 8 shows the distribution of the core-swirl ratio $\beta$ along the dimensionless radius for the 9 test cases. The experimental results and those obtained by the RSM and $k-\omega$ SST models are compared to the analytical law of Daily et al. [12] (Eq.3) and to the ones obtained by Poncet 
et al. $[7,13]$ for Batchelor (Eq.4) and Stewartson (Eq.5) flows.

In Test cases $1-3$, the RSM model predicts Stewarston flows with a core-swirl ratio close to zero even if a small increase can be noticed towards the periphery of the cavity. It is good agreement with the two analytical laws dedicated to this flow regime. The mean flow is thus clearly dominated by the impinging jet $(0.065 \leq N \leq 0.162)$ and rotation effects remain weak, which explains why the analytical law of Eq. (4) is not appropriated. For these test cases and in certain flow regions, measured velocities are of the same order than the measurement uncertainty and are very fluctuating when non-dimensionalized so the analysis is biased. The relative high values reached by $\beta$ for Test case 2 and $r / R_{e x t}>0.7$ may be attributed to peripheral effects and some possible ingress of fluid.

By increasing the rotation rate (and so $N$ and $R e$ ), the core-swirl ratio increases as expected. For Test cases $4-6$, one can observe from the radial distributions of $\beta$ the transition from the Stewartson flow structure obtained for $r<r_{c}$, where the predominant effect is due to the impinging jet, to a Batchelor flow structure at $r>r_{c}$ where rotation effects become not negligible. For example, for Test case 5 , it is found that $r_{c}=0.66 R_{e x t}$ from the PIV measurements. A quite good agreement between the experiments and the numerics is here obtained with the same radial evolution of $\beta$. It was indeed expected that the RSM performs better for higher rotational Reynolds numbers Re as already observed in enclosed rotor-stator cavities by Elena and Schiestel [1]. At low Re, such that for Test cases $1-3$, the RSM may have the tendency to relaminarize the flow and predicts laminar flows, which are known to exhibit smaller values of the core-swirl coefficient than in the turbulent regime (see for example in $[10,32]$ ). On the contrary, the $k-\omega$ SST overpredicts the core-swirl ratio especially for Test case 4 and for low radii in the two other cases.

If one increases further $R e$ up to $5.32 \times 10^{5}$ (Test cases $7-9$ ), the flow structure switches to a Batchelor flow at lower radii than in the previous cases, at around $r_{c} \simeq 0.5 R_{e x t}$ in Test case 7 for example. This critical radius for the transition from the Stewartson to the Batchelor flow regime increases when one increases the jet Reynolds number $R e_{j}$ to reach $r_{c} \simeq 0.65 R_{\text {ext }}$ in Test case 9 . The experimental data appear to be in particular good agreement with the values predicted by Eq. (3) and (5).

Figure 9 shows the mean radial velocity distributions, normalized by the jet velocity $V$, along the dimensionless radius $r / R_{\text {ext }}$ for Test case $5(N=0.866$, see Table 1$)$ at three axial positions. One position is located close to the rotor $(z / e=0.23)$, one is around mid-gap $(z / e=0.53)$ and one is near the stator $(z / e=0.84)$. The present results are compared to the LDA measurements of Mingawa and Obi [19] and the numerical results of Manceau et al. [39] obtained by an EB-RSM model in the case of a jet impinging onto a rotating disk. Whatever the axial position, the present measurements provide negative radial velocities for $r / R_{\text {ext }} \leq 0.16(r / D \leq 2)$, meaning that the flow is mainly centripetal. It was thought that the flow should be centrifugal due to 
the presence of the jet, so it is identified as a recirculation area. Its thickness is very large because negative radial velocities are observed near the stator but also near the rotor. It must involve very high radial velocities in the rotor boundary layer. At larger radii $\left(r / R_{e x t} \geq 0.16\right.$ or $\left.r / D \geq 2\right)$, radial velocities increase and reach a maximum at around $r / R_{\text {ext }} \simeq 0.26(r / D \approx 3)$ before a slow decrease until the air gap outlet. As expected, the radial velocities near the rotor are higher than near the stator.

A good agreement is obtained between the PIV measurements and the RANS results at large radii $r / R_{e x t} \geq 0.25$, where $V_{r}$ remains quite weak. On the other hand, large differences can be observed in the jet dominated area. These discrepancies may be explained as following. After impinging on the rotor, the jet deflects in radial direction. A radial wall jet begins to form and creates a thin wall boundary layer. Hadžiabdić and Hanjalić [40] showed the existence of vortical structures formed at the wall-jet edge and counter-rotating secondary vortices created near the impinging plane (rotor plane in our case). The secondary vortices rolled up between the impinging plane and the vortical structures at the wall-jet edge. Footprints of these structures were displayed in the POD analysis of Nguyen et al. [26]. However, in the PIV measurements, the laser thickness was $1 \mathrm{~mm}$, which is not small enough compared to the boundary layer thickness and the small gap between the rotor and the stator. Velocity measurements on the laser planes could be the volume-averaged values of particle displacements that displaying the entrainment of the vortical structures around the stagnation point in the radial direction. An investigation of the flow structures near the impinging jet in the $(r, z)$ plane using stereo PIV is arranged for a further study and validation of the numerical simulation.

The RSM profiles very close to the impinging zone $\left(r / R_{e x t} \leq 0.05\right.$, on the rotor side) are thus in better agreement with the previous results of $[19,39]$ obtained without the stator. The RSM predicts the centripetal flow along the stator but the size of this recirculation is much weaker than the measured one. When one approaches the rotating disk, the values of the mean radial velocity increase to reach the peak value obtained previously by the EB-RSM model of Manceau et al. [39] at $r / R_{e x t} \simeq 0.075(r / D \simeq 0.9)$. It is noticeable that almost the same distributions (not shown here) have been obtained for the other sets of parameters.

The $k-\omega$ SST predicts rather the same profiles as the ones obtained by the RSM. The radial velocity is positive at low radii after the jet impingement with peak values much higher than those obtained by the RSM. Then, $V_{r}$ tends to zero along the rotor, also around $r / R_{\text {ext }} \simeq 0.25$. The discrepancies may be explained by the larger recirculation areas previously evoked in Figure 6. It is noticeable that it reproduces quite well the radial velocity profile on the stator side.

Those observations about radial and tangential mean velocity components are concordant with those by Poncet et al. [7]. The flow is at very low radii, near the jet impingement, mainly centrifugal. Thus, when increasing radius, radial 
velocities tend to decrease and rotation effects become dominant inside the air-gap. If the jet Reynolds number is too low compared to the rotational Reynolds number, a centripetal flow can occur near the stator at outer radii and the flow tends to become a Batchelor type flow. Nevertheless, in this case, the rotating core rotates at a lower speed than in the rotor-stator configuration without superposed flow $(\beta=0.438)$, which is expected in unshrouded system [9].

\subsection{Turbulent statistics}

Figure 10 presents some maps of the turbulence kinetic energy $k$ (normalized by its maximum) in a meridian plane for Test Cases 4,5 and 6 obtained by the $\mathrm{RSM}$ and the $k-\omega$ SST models. The rotation is then fixed to $R e=2.66 \times 10^{5}$ and the jet axial Reynolds number is increased from $R e_{j}=17200$ to 43000 . Both models exhibit the same behavior. Turbulence is mainly produced by the impinging jet at low radii, where the lowest values of the local Reynolds number $R e_{r}$ are observed. Rotation has then only a weak effect on the turbulence production. Not surprisingly, turbulence kinetic energy increases for increasing values of $R e_{j}$. The cavity being opened to atmosphere, the fluid at the periphery does not flow inwards along the stationary disk and then, there is no convective transport of turbulence close to the rotation axis as reported by Cheah et al. [41] in a enclosed rotor-stator system.

To have a more precise view of the turbulent intensities for Test case 5, the radial distributions of the three Reynolds stress tensor components available in the experiments are plotted in Figure 11 at three axial positions and compared to the LDA measurements of Mingawa and Obi [19] and the numerical results of Manceau et al. [39] obtained by an EB-RSM model in the case of a jet impinging onto a rotating disk. The two normal components increase up to a peak value reached at around $r / R_{\text {ext }} \simeq 0.12(r / D \simeq 1.4)$. Then, they decrease towards the periphery of the cavity. The peak values are particularly well predicted by the RSM. On the other hand, the RSM seems to relaminarize the flow more rapidly than expected in the experiments. Compared to the former results $[19,39]$, the stator does not affect so much the behavior of the radial normal Reynolds stress tensor component. The cross component has a more singular behavior: after reaching a maximum around $r / R_{\text {ext }} \simeq 0.15$, it decreases with the radius but can become negative close to the stator, which is not predicted here by the RSM. It is important to note that, for the three Reynolds stresses considered here, turbulent intensities are more important on the rotor side and at given radius, decrease when one approaches the stationary disk. The same tendencies have been obtained for the other test cases (not shown here), $R e$ and $R e_{j}$ having only a slight effect on the distributions of the turbulent intensities. Comparing the two RANS modelings, the tendencies are rather the same with higher turbulence intensities close to the jet and 
peak values observed along the rotor. Nevertheless, the $k-\omega$ SST strongly overpredicts the turbulence intensities in the jet impingement zone.

Figure 12 shows anisotropy invariant maps (the componentality as defined by Reynolds [43]) for the Reynolds stress tensor at three radial positions for Test case 5 . The second $A_{2}$ and third $A_{3}$ invariants of the anisotropy tensor $a_{i j}$ of the second moments of the fluctuations are defined as: $A_{2}=a_{i j} a_{j i}$ and $A_{3}=a_{i j} a_{j k} a_{k i}$. The results of the RSM model respect the realizability diagram of Lumley [42] as they remain within the region delimited by the two lines corresponding to the axisymmetric flow $A_{3}= \pm A_{2}^{3 / 2} / \sqrt{6}$ and the straight upper one corresponding to the two-component limit $A_{3}=A_{2}-8 / 9$. The three radial positions considered correspond to three distinct flow regions. At $r / R_{e x t}=0.1$ (Fig.12a), the flow is dominated by the impinging jet and exhibits a Stewartson flow structure for the three test cases considered here. In that flow region, turbulence exhibits a three-component behavior. At mid-gap, it tends to the isotropic limit $\left(A_{2}=A_{3}=0\right)$. It confirms the previous results of Haddadi and Poncet [5] for torsional Couette flows in a shrouded rotor-stator cavity. At $r / R_{\text {ext }}=0.8$ (Fig.12c), where the flow has switched to the Batchelor flow structure $(\beta>0.1$, Fig.8) and is mainly dominated by rotation, turbulence remains at three components. At $r / R_{\text {ext }}=0.4$ (Fig.12b), both effects, rotation and jet impingement, live together. In that case, turbulence is isotropic at midgap and tends to the one-component limit (high values of $A_{2}$ and $A_{3}$ ) close to the rotor. Similiar behaviors are observed whatever the test case, which means that the flow anisotropy depends only weakly on the rotational and jet Reynolds numbers in this range of parameters.

Note that the structural anisotropy or the dimensionality as defined by Reynolds [43] is here axisymmetric by whatever the flow conditions. This is inherent to the choice of the dimensionality tensor in the RSM model made by Elena and Schiestel [32].

\section{Convective Heat Transfer}

Results are discussed in terms of the local Nusselt number defined as: $N u=$ $\frac{D J_{w}}{\rho C_{p} \alpha\left(T_{\text {rotor }}-T_{\infty}\right)}$, where $D$ is the jet diameter, $\rho$ the density of air and $C_{p}$ its specific heat capacity. $J_{w}$ is the wall heat flux given by: $J_{w}=-\left.\rho C_{p} \alpha \frac{\partial T}{\partial z}\right|_{w}$, where the index $w$ denotes a value evaluated at the wall.

Figure 13 shows the local Nusselt number, calculated with the jet diameter $D$ as reference length, along the dimensionless radius $r / R_{\text {ext }}$ for 3 rotational Reynolds numbers and 2 jet Reynolds numbers. In all cases, $N u$ is a decreasing function with $r / R_{e x t}$, the highest values being observed near the jet. Data for $r / R_{\text {ext }}<0.1$ are not available due to the fact that the pipe which brings the jet do not allow an optical access to this area.

For a fixed jet Reynolds number $R e_{j}$, considering Figures $13 \mathrm{a}$ and $\mathrm{b}$, the 3 
profiles relative to the 3 rotational Reynolds numbers show that the local Nusselt number does not depend on $R e$ for $r / R_{\text {ext }} \leq\left(r / R_{\text {ext }}\right)_{0} .\left(r / R_{\text {ext }}\right)_{0}$ is equal to around 0.34 for $R e_{j}=1.6 \times 10^{4}$ and 0.5 for $R e_{j}=4.2 \times 10^{4}$. In this region, it is assumed that the convective heat transfer are the same as if there was no rotation. By comparing the two figures, it can be seen also that the local Nusselt number increases with the jet Reynolds number. Thus, when $r / R_{e x t} \leq\left(r / R_{e x t}\right)_{0}$ and for all tested $R e$ and $R e_{j}$ values, radial velocities are very high in a very thin rotor boundary layer because the flow is centripetal everywhere outside this boundary layer. In this area, the flow is not rotating so the rotational Reynolds number $R e$ is not an influent parameter. Those high centrifugal velocities involve strong shear stresses and so high heat transfer. As the size of this area is the same for all $R e_{j}$, increasing the jet velocity results in an increase in the convective heat transfer.

After that, one can detect another critical radius $\left(r / R_{\text {ext }}\right)_{1}$ where the curves relative to $R e=3 \times 10^{4}$ and $R e=2.58 \times 10^{5}$ separate. It occurs at $\left(r / R_{\text {ext }}\right)_{1}=$ 0.67 for $R e_{j}=1.6 \times 10^{4}$ and at $\left(r / R_{e x t}\right)_{1}=0.77$ for $R e_{j}=4.2 \times 10^{4}$. The influence of rotation on the convective heat transfer appears after a critical radius which is lower when increasing $R e$ and higher when increasing $R e_{j}$. The decrease of the local Nusselt number when $r / R_{e x t}$ is increasing is due to the thickening of the rotor boundary layer associated with lower radial velocities and higher tangential velocities. After $\left(r / R_{e x t}\right)_{1}$, the local Nusselt number continues to decrease but more slowly than near the center of the rotor. One can observe that it remains quite constant in the case where $R e_{j}=1.6 \times 10^{4}$ and $R e=2.58 \times 10^{5}$. Local Nusselt numbers, which are reached at outer radii, increase with the rotational speed, as already observed by Manceau et al. [39] for an impinging jet onto a rotating disk without the confinement stator.

On Figure 13a, results from Katti and Prabhu [44] are also reported. They have been established for $R e_{j}=16000, e / D=0.5$ and without rotation nor confinement. They are available for $r / R_{\text {ext }} \leq 0.5$ where one proved before that the rotation has no high effect. The present results are concordant with those of Katti and Prabhu [44] with slightly lower values. This difference may be attributed to confinement effects as already demonstrated by Gao and Ewing $[17]$.

As mentioned above, the local Nusselt number also increases with the jet Reynolds number, which can be seen by comparing Figures $13 \mathrm{a}$ and b. However, at the same value of $R e$, the difference between both $R e_{j}$ is large near the impingement point and decreases as the radius increases, meaning that the influence of the jet Reynolds number seems to disappear at the periphery of the cavity (large $r / R_{\text {ext }}$ ).

The RSM and $k-\omega$ SST models provide very satisfactory results for Test cases 4 and 6 in terms of the distribution of the local Nusselt number. Whatever the jet Reynolds number, similar behaviors are observed: $N u$ decreases more rapidly with the local radius in the computations before being rather constant at large radii where rotation effects dominate. The RSM seems to offer the best results, especially for the peak value in case 6 , where the $k-\omega$ 
SST slightly underestimates the heat transfer coefficient.

For engineering applications, it can be also useful to consider the averaged Nusselt number $N u_{a v}^{D}$ based on the jet diameter $D$. Experimental results are typically correlated by the form [45]:

$$
N u_{a v}=a \operatorname{Re}_{j}^{n} \operatorname{Pr}^{l} f(e / D)
$$

where $f(e / D)$ is an empirically determined function and $a, n$ and $l$ are constants determined by experiments. Additional dimensionless parameters may be added to the correlation to account for other effects (angle of incidence, surface curvature, pressure loss ....). When rotation effects are superimposed on the jet flow problem, one can also suppose that $N u_{a v}$ depends also on the rotational Reynolds number Re to a power $m$. For turbulent rotor-stator flows without jet, it is quite classical to obtain $m=0.8$ [10]. Figure 14 presents the dependence of $N u_{a v}^{D}$ on $R e$ for two values of the jet Reynolds number $R e_{j}$. The averaged Nusselt number is so an increasing function with $R e$ and $R e_{j}$, which is concordant with the observations by Sara et al. [18] obtained for lower Re and larger jet aspect ratios. From the test cases considered here but also from other measurements on the same set-up, one can correlate the experimental values under the form:

$$
N u_{a v}=N u_{0}+C \times R e^{m}
$$

where $C=2 \times 10^{-4}$ and $N u_{0}$ is the averaged Nusselt number obtained without rotation. This value can be deduced from the experiments performed by Sagot et al. [46] in the case of a round jet impinging a circular flat stationary plate at constant temperature. They provided the following correlation valid for $10^{4}<R e_{j}<3 \times 10^{4}, 2<e / D<6$ and $3<R_{e x t} / D<10$ :

$$
N u_{a v}=0.0622 R e_{j}^{0.8}\left(1-0.168 \frac{R_{e x t}}{D}+0.008\left(\frac{R_{e x t}}{D}\right)^{2}\right)\left(\frac{e}{D}\right)^{-0.037}
$$

From our measurements, $N u_{0}$ is equal to 18 and 36 for $R e_{j}=1.6 \times 10^{4}$ and $4.2 \times 10^{4}$ respectively to be compared with the values 20.3 and 44 deduced from Equation 22 . The weak discrepancies may be attributed to the very small value of $e / D=0.24$ (not in the validity domain $2<e / D<6$ ) and due to the difference of boundary conditions applied on the rotor (wall heat flux against imposed wall temperature). The exponent $m=0.85$ highlighting the dependance of $N u_{a v}$ on the rotational Reynolds number is in good agreement with the common value $m=0.8$ found in many rotating disk systems [10]. It is more adventurous to get the coefficient $n$ as only two values of the jet Reynolds number $R e_{j}$ are considered here. Nevertheless, one can propose $n=0.72$, which falls between the values obtained by Wen and Jang [47] $(n=0.696)$ 
or Tawfek [48] ( $n=0.691)$ for a round jet impinging a flat surface and the value $(n=0.8)$ from Equation (22). It is noteworthy that $N u_{a v}^{D}$ does not scale neither with the turbulent flow parameter $\lambda_{t}$ nor with the rotation parameter $N$.

The averaged Nusselt numbers have also been computed by the RSM and $k-\omega$ SST models for Test cases 4 and 6 . As it can be seen in Table 2, the RSM results are in excellent agreement with the values obtained experimentally, whereas the $k-\omega$ SST slightly underpredicts the averaged Nusselt number in both cases.

\section{Conclusion}

In this study, experimental measurements of flow structure, mean flow field, turbulence statistics and heat transfer coefficients have been performed in an unshrouded rotor-stator system with a jet impingement. Some comparisons with new predictions of the Reynolds Stress Model of Elena and Schiestel [1] and of a $k-\omega$ SST model have been provided. The interdisk spacing and the jet aspect ratio are kept very small so that the stator influences the flow within the gap. PIV measurements have been performed for 3 axial planes inside the air-gap, giving radial and tangential velocities and the corresponding Reynolds stress tensor components along the whole rotor diameter. Influences of rotation and jet velocity have been highlighted and compared with convective heat transfer over the rotor, determined with the use of infrared thermography.

Three different flow regions have been identified when increasing the radius:

(1) a recirculation zone characterized by high heat transfer and high turbulence intensities develops near the jet impingement;

(2) a mixed region at intermediate radii where rotational and jet effects coexist. Turulence intensities and the heat transfer coefficient along the rotor decrease when moving radially outwards.

(3) a zone located at the periphery of the cavity: rotational effects dominate and leads to higher heat transfer than without rotation. For some sets of parameters, flow ingress has been observed along the stator leading to a flow structure with unmerged boundary layers.

The RSM model compares relatively well with the experimental data especially in terms of turbulence intensities and heat transfer coefficient, with some discrepancies on the jet width. If the $k-\omega$ SST model strongly overestimates the peak values of the Reynolds stresses, it reproduces the jet width at low radii and provides the good trend for the distribution of the heat transfer coefficient over the rotor with slighlty underestimated values. The size of the recirculation zone appearing at low radii along the stator seems to be slightly better modeled by the RSM. 
The experimental technique used in the present study does not allow the access to the data in the vicinity of the rotating disk. To improve our knowledge, it would be very interesting to investigate the flow field and the turbulence level inside the rotor boundary layer. It will be next realized with stereo-PIV made in a $(r, z)$ plane. From a numerical point of view, three-dimensional calculations using Large Eddy Simulation are now required to investigate in more details the three-dimensional nature of the turbulent boundary layers developed along both disks.

\section{Acknowledgement}

This work was undertaken as part of the MEDEE (Energy control of electric devices) program. The authors would like to thank the Nord-Pas-De-Calais region and the EU (FEDER) for their financial support as well as the LABEX MEC (ANR-11-LABX-0092) through the HYDREX program.

\section{References}

[1] Elena, L., and Schiestel, R., 1996. "Turbulence modeling of rotating confined flows". Int. J. Heat Fluid Flow, 17, pp. 283-289.

[2] Daily, J., and Nece, R., 1960. "Chamber dimension effects on induced flow and frictional resitance of enclosed rotating disks". J. Basic Eng., 82, pp. 217-232.

[3] Pellé, J., and Harmand, S., 2007. "Heat transfer measurements in an opened rotor-stator system air-gap". Exp. Thermal Fluid Sci., 31(3), pp. 165-180.

[4] Andersson, H., and Lygren, M., 2006. "LES of open rotor-stator flow". Int. J. Heat Fluid Flow, 27(4), pp. 551-557.

[5] Haddadi, S., and Poncet, S., 2008. "Turbulence modeling of torsional Couette flows". Int. J. Rotating Machinery, Article ID 635138.

[6] Batchelor, G., 1951. "Note on a class of solutions of the Navier-Stokes equations representing steady rotationally-symmetric flow". Quart. J. Mech. Appl. Math., 4(1), pp. 29-41.

[7] Poncet, S., Chauve, M., and Schiestel, R., 2005. "Batchelor versus Stewartson flow structures in a rotor-stator cavity with throughflow". Phys. Fluids, 17(075110).

[8] Stewartson, K., 1953. "On the flow between two rotating coaxial disks". In Proc. Camb. Phil. Soc., Vol. 49, Cambridge University Press, pp. 333-341.

[9] Brady, J., and Durlofsky, L., 1987. "On rotating disk flow". J. Fluid Mech., 175, pp. 363-94. 
[10] Owen, J., and Rogers, R., 1989. Flow and heat transfer in rotating-disc systems, Vol. 1: Rotor-Stator Systems. John Wiley \& Sons Inc., New-York.

[11] Kreith, F., Doughman, E., and Kozlowski, H., 1963. "Mass and heat transfer from an enclosed rotating disk with and without source flow". J. Heat Transfer, 85, pp. 153-163.

[12] Daily, J., Ernst, W., and Asbedian, V., 1964. Enclosed rotating disks with superposed throughflow. Tech. Rep. 64, M.I.T, Department of Civil Engineering.

[13] Poncet, S., Schiestel, R., and Chauve, M., 2005. "Centrifugal flow in a rotorstator cavity". J. Fluid Eng., 127(4), pp. 687-694.

[14] Childs, P., 2011. Rotating Flow. Elsevier, Butterworth-Heinemann, New-York.

[15] Da Soghe, R., Facchini, B., Innocenti, L., and Micio, M., 2012. "Some improvements in a gas turbine stator-rotor systems core-swirl ratio correlation". International Journal of Rotating Machinery.

[16] Owen, J., Haynes, C., and Bayley, F., 1974. "Heat transfer from an air-cooled rotating disk". Proc. Roy. Soc. A, 336, pp. 453-473.

[17] Gao, N., and Ewing, D., 2006. "Investigation of the effect of confinement on the heat transfer to round impinging jets exiting a long pipe". Int. J. Heat Fluid Flow, 27(1), pp. 33-41.

[18] Sara, O., Erkmen, J., Yapici, S., and Çopur, M., 2008. "Electrochemical mass transfer between an impinging jet and a rotating disk in a confined system". Int. Comm. Heat Mass Transfer, 35(3), pp. 289-298.

[19] Minagawa, Y., and Obi, S., 2004. "Development of turbulent impinging jet on a rotating disk". Int. J. Heat Fluid Flow, 25(5), pp. 759-766.

[20] Popiel, C., and Boguslawski, L., 1986. "Local heat transfer from a rotating disk in an impinging round jet". J. Heat Transfer, 108, pp. 357-364.

[21] Chen, Y., Lee, W., and Wu, S., 1998. "Heat (mass) transfer between an impinging jet and a rotating disk". Heat Mass Transfer, 34(2), pp. 195-201.

[22] Pellé, J., and Harmand, S., 2009. "Heat transfer study in a rotor-stator system air-gap with an axial inflow". Appl. Therm. Eng., 29(8-9), pp. 1532-1543.

[23] Harmand, S., Pellé, J., Poncet, S., and Shevchuk, I., 2013. "Review of fluid flow and convective heat transfer within rotating disk cavities with impinging jet". Int. J. Therm. Sci., 67, pp. 1-30.

[24] Launder, B., Poncet, S., and Serre, E., 2010. "Laminar, transitional and turbulent rotor-stator flows". Annual Review of Fluid Mechanics, 42, pp. 229248.

[25] Shevchuk, I., 2009. Convective heat and mass transfer in rotating disk systems, Vol. 45. Springer Verlag, Berlin. 
[26] Nguyen, N., Pellé, J., Harmand, S., and Poncet, S., 2012. "PIV measurements of an air jet impinging on an open rotor-stator system". Exp. Fluids, 53, p. 401412.

[27] Kompenhans, J., Raffel, M., Wereley, S., and Willert, C., 2007. Particle image velocimetry: a practical guide; with 42 tables. Springer.

[28] Westerweel, J., 1994. "Efficient detection of spurious vectors in particle image velocimetry data". Exp. Fluids, 16(3), pp. 236-247.

[29] Coleman, H., and Steele, W., 1995. "Engineering application of experimental uncertainty analysis". AIAA journal, 33(10), pp. 1888-1896.

[30] Pellé, J., and Harmand, S., 2011. "Convective heat transfer in a rotor stator system air gap with multiple suctions of fluid through the stator". J. Heat Transfer, 133, p. 071707.

[31] Dorfman, L., and Kemmer, N., 1963. Hydrodynamic resistance and the heat loss of rotating solids. Oliver \& Boyd, Edinburgh.

[32] Poncet, S., 2005. "écoulements de type rotor-stator soumis à un flux: de Batchelor à Stewartson". PhD thesis, Université Aix-Marseille I.

[33] Poncet, S., Haddadi, S., and Viazzo, S., 2011. "Numerical modeling of fluid flow and heat transfer in a narrow Taylor-Couette-Poiseuille system". Int. J. Heat Fluid Flow, 32, pp. 128-144.

[34] Poncet, S., and Schiestel, R., 2007. "Numerical modeling of heat transfer and fluid flow in rotor-stator cavities with throughflow". Int. J. Heat Mass Transfer, 50(7-8), pp. 1528-1544.

[35] Launder, B., and Tselepidakis, D., 1994. "Application of a new second-moment closure to turbulent channel flow rotating in orthogonal mode". Int. J. Heat Fluid Flow, 15(1), pp. 2-10.

[36] Da Soghe, R., Facchini, B., Innocenti, L., Andreini, A., and Poncet, S., 2010. "Numerical benchmark of turbulence modeling in gas turbine rotor-stator system". Proceedings of ASME TurboExpo, GT2010-22627.

[37] Smirnov, P.E., and Menter, F.R., 2008. "Sensitization of the SST turbulence model to rotation and curvature by applying the Spalart-Shur correction term". Proceedings of ASME TurboExpo, GT2008-50480.

[38] Spalart, P.R., and Shur, M.L., 1997. "On the sensitization of turbulence models to rotation and curvature". Aerospace Science and Technology, 1(5), pp. 297302.

[39] Manceau, R., Perrin, R., Hadziabdic, M., Fourment, P., and Benhamadouche, S., 2009. "Turbulent jet impinging onto a rotating disc: a collaborative evaluation of rans models". Y. N. K. Hanjalic and S. Jakirlic, eds., Turbulence, Heat and Mass Transfer 6, Begell House.

[40] Hadžiabdić, M., and Hanjalić, K., 2008. "Vortical structures and heat transfer in a round impinging jet". J. Fluid Mech., 596, pp. 221-260. 
[41] Cheah, S., Iacovides, H., Jackson, D., Ji, H., and Launder, B., 1994. "Experimental investigation of enclosed rotor-sator disk flows". Exp.Thermal Fluid Sci., 9, pp. 445-455.

[42] Lumley, J., 1978. "Computational modeling of turbulent flows". Adv. Appl. Mech., 18, pp. 123-176.

[43] Reynolds, W.C., 1991. "Towards a structure-based turbulence model". T.B. Gatski, S. Sarkar, and C.G. Speziale, eds., Studies in Turbulence, SpringerVerlag.

[44] Katti, V., and Prabhu, S., 2008. "Experimental study and theoretical analysis of local heat transfer distribution between smooth flat surface and impinging air jet from a circular straight pipe nozzle". Int. J. Heat Mass Transfer, 51(17-18), pp. $4480-4495$.

[45] Zuckerman, N., and Lior, N., 2006. "Jet impingement heat transfer: Physics, correlations, and numerical modeling". Advances in Heat Transfer, 39, pp. 565631.

[46] Sagot, B., Antonini, G., Christgen, A., and Buron, F., 2008. "Jet impingement heat transfer on a flat plate at a constant wall temperature". Int. J. Thermal Sci., 47(12), pp. 1610-1619.

[47] Wen, M.-Y., and Jang, K.-J., 2003. "An impingement cooling on a flat surface by using circular jet with longitudinal swirling strips". Int. J. Heat Mass Transfer, 46, pp. 4657-4667.

[48] Tawfek, A. A., 1996. "Heat transfer and pressure distributions of an impinging jet on a flat surface". Heat Mass Transfer, 32, pp. 49-54. 
- Table 1: Flow control parameters for the 9 test cases.

- Table 2: Comparisons for the averaged Nusselt number. 
Table 1

Poncet et al., submitted to Int. J. Heat Fluid Flow.

\begin{tabular}{|c|c|c|c|c|c|c|c|}
\hline Test case & $\omega R_{\text {ext }}(\mathrm{m} / \mathrm{s})$ & $R e\left(\times 10^{-5}\right)$ & $V(\mathrm{~m} / \mathrm{s})$ & $R e_{j}\left(\times 10^{-3}\right)$ & $C_{w}$ & $\lambda_{t}$ & $N$ \\
\hline \hline 1 & 1.623 & 0.33 & 10 & 17.2 & 1133 & 0.275 & 0.162 \\
\hline 2 & 1.623 & 0.33 & 15 & 25.8 & 1700 & 0.413 & 0.108 \\
\hline 3 & 1.623 & 0.33 & 25 & 43 & 2833 & 0.688 & 0.065 \\
\hline 4 & 12.985 & 2.66 & 10 & 17.2 & 1133 & 0.052 & 1.299 \\
\hline 5 & 12.985 & 2.66 & 15 & 25.8 & 1700 & 0.078 & 0.866 \\
\hline 6 & 12.985 & 2.66 & 25 & 43 & 2833 & 0.13 & 0.519 \\
\hline 7 & 25.971 & 5.32 & 10 & 17.2 & 1133 & 0.03 & 2.597 \\
\hline 8 & 25.971 & 5.32 & 15 & 25.8 & 1700 & 0.045 & 1.731 \\
\hline 9 & 25.971 & 5.32 & 25 & 43 & 2833 & 0.074 & 1.039 \\
\hline
\end{tabular}


Table 2

Poncet et al., submitted to Int. J. Heat Fluid Flow.

\begin{tabular}{|c|c|c|c|}
\hline Test case & Experiments & RSM & k- $\omega$ SST \\
\hline \hline 4 & 26.64 & 29.79 & 18.5 \\
\hline 6 & 42.65 & 40.54 & 32.8 \\
\hline
\end{tabular}


- Fig. 1: Experimental set-up.

- Fig. 2: PIV measurement system.

- Fig. 3: Distribution of the local Nusselt number as a function of the local Reynolds number $R e_{r}=R e \times\left(r / R_{e x t}\right)^{2}$ for $R e=5.16 \times 10^{5}$. Comparison with the correlations obtained by Dorfman [31].

- Fig. 4: Axial profiles of the mean radial and tangential velocity components and the corresponding Reynolds stress tensor components at $r / R_{\text {ext }}=0.56$ and $R e=1.04 \times 10^{6}$ in a shrouded rotor-stator cavity of aspect ratio $G=0.036$ : (a) $C_{w}=0$, (b) $C_{w}=1976$, (c) $C_{w}=5929$, (d) $C_{w}=9881$. Comparisons between the present RSM (straight lines), a low-Reynolds number $k-\epsilon$ model (dashed lines) and the LDV measurements (symbols) of Poncet [32].

- Fig. 5: Streamline patterns obtained by the RSM, from (a) Test case 1 to (i) Test case 9. Apparent aspect ratio equal to 0.07.

- Fig. 6: Streamline patterns obtained by the $k-\omega$ SST, for Test cases (a) 4, (b) 5 and (c) 6. Apparent aspect ratio equal to 0.07.

- Fig. 7: Normalized velocity vectors in the plane $(x, y)$ for Test cases $1(\mathrm{a}, \mathrm{b})$ and $7(\mathrm{c}, \mathrm{d})$, in two planes, near the rotor $(\mathrm{a}, \mathrm{c})$ and near the stator $(\mathrm{b}, \mathrm{d})$.

- Fig. 8: Radial variation of the core-swirl ratio $\beta$ for the 9 test cases: (a) test case $1 \ldots$ (i) test case 9 . Comparisons between the experiments (symbols), the RSM (solid black lines), the k- $\omega$ SST model (dashed black lines) and the Equations $(3,4,5)$.

- Fig. 9: Radial variation of the averaged mean radial velocity $V_{r} / V$ for Test case 5 and three axial positions: one near the rotor $(z / e=0.23)$, at mid-gap $(z / e=0.53)$ and one close to the stator $(z / e=0.84)$. Comparisons between the experiments (symbols), the RSM (dashed lines), the $k-\omega$ SST (solid lines), the LDA measurements of Mingawa and Obi [19] (green squares) and the EB-RSM model of Manceau et al. [39] (dash-dotted line).

- Fig. 10: Maps of the turbulence kinetic energy $k$ normalized by its maximum value for Test Case 4 (a,b), 5 (c,d) and 6 (e,f) obtained by the RSM (a,c,e) and the $k-\omega$ SSt model $(\mathrm{b}, \mathrm{d}, \mathrm{f})$. Apparent aspect ratio of the cavity equal to 0.07 .

- Fig. 11: Radial variation of three components of the Reynolds stress tensor for Test case 5 and at three axial positions: one near the rotor $(z / e=$ $0.23)$, at mid-gap $(z / e=0.53)$ and one close to the stator $(z / e=0.84)$. Comparisons between the experiments (symbols), the RSM (dashed lines), the $k-\omega \mathrm{SST}$ (solid lines), the LDA measurements of Mingawa and Obi [19] (green squares) and the EB-RSM model of Manceau et al. [39] (dash-dotted line). Note that the Reynolds stress tensor components are here normalized as follows: $R_{r r}^{*}=\overline{v_{r}^{\prime 2}} / V^{2}, R_{\theta \theta}^{*}=\overline{v_{\theta}^{\prime 2}} / V^{2}$ and $R_{r \theta}^{*}=\overline{v_{r}^{\prime} v_{\theta}^{\prime}} / V^{2}$.

- Fig. 12: Anisotropy invariant maps obtained by the RSM for Test case 5 at three characteristic radial positions: (a) $r / R_{\text {ext }}=0.1$, (b) $r / R_{\text {ext }}=0.4$ and (c) $r / R_{\text {ext }}=0.8$.

- Fig. 13: Radial profiles of local Nusselt number: (a) $R e_{j}=16000$, (b) $R e_{j}=$ 
42000.

- Fig. 14: Variations of the averaged Nusselt number $N u_{a v}^{D}$ (based on the jet diameter $D$ ) along the rotor against the rotational Reynolds number for two values of the jet Reynolds number $R e_{j}=16000$ and $R e_{j}=42000$. Comparison with correlation laws. 


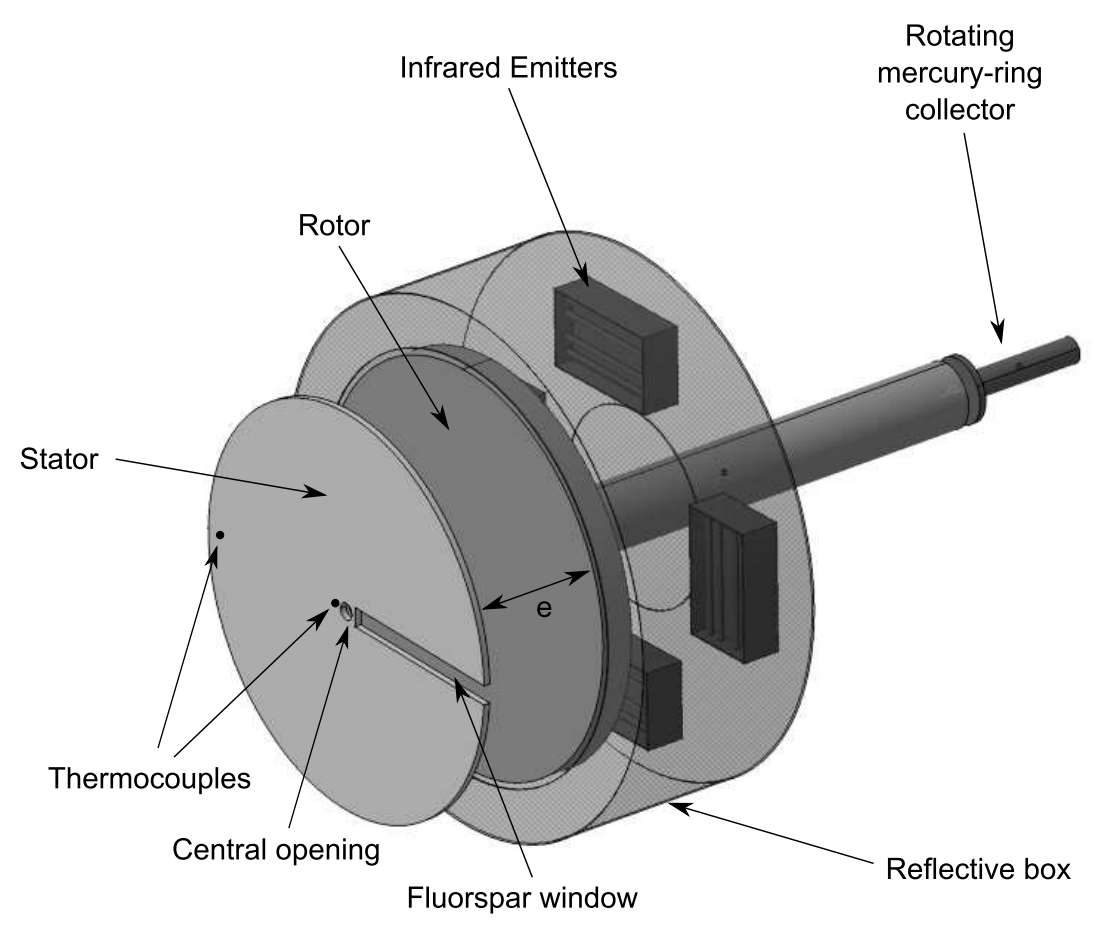

Fig. 1. Poncet et al., submitted to Int. J. Heat Fluid Flow. 


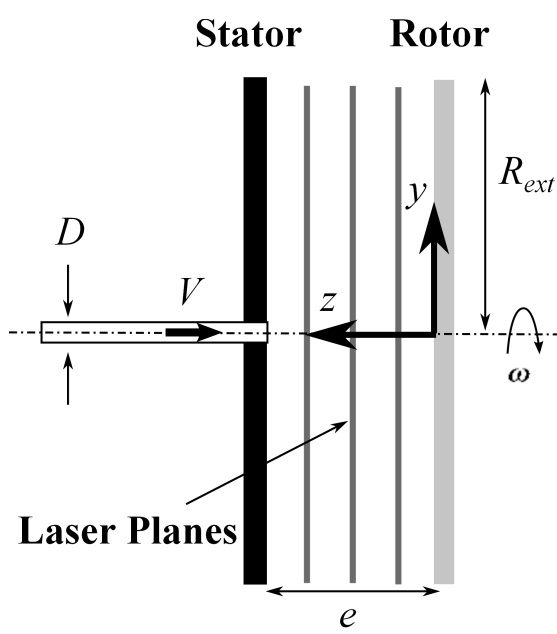

Side View

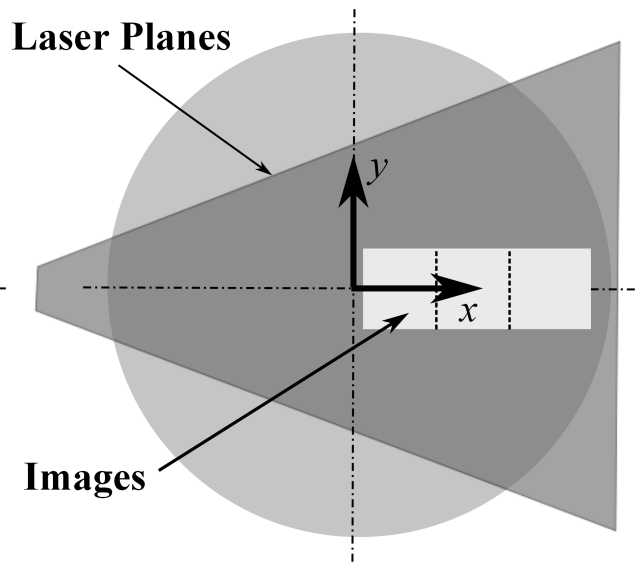

Front view

Fig. 2. Poncet et al., submitted to Int. J. Heat Fluid Flow. 


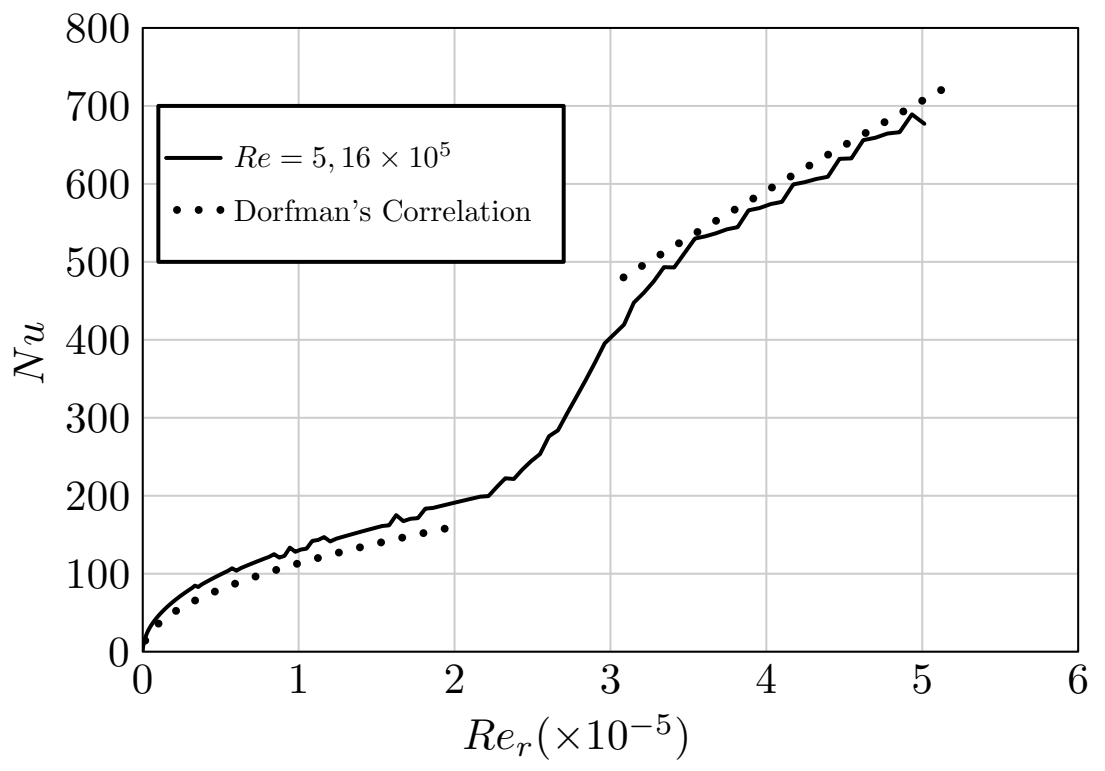

Fig. 3. Poncet et al., submitted to Int. J. Heat Fluid Flow. 
(a)
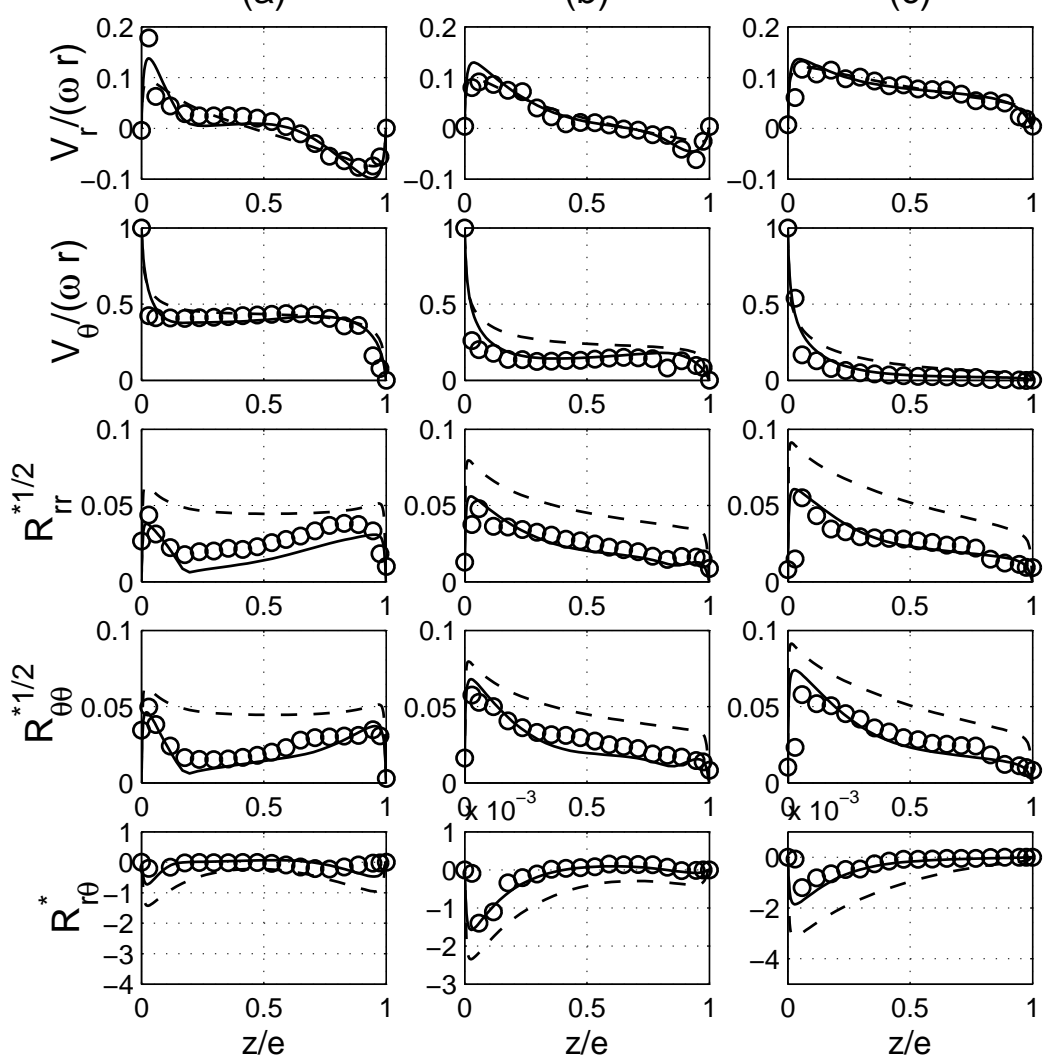

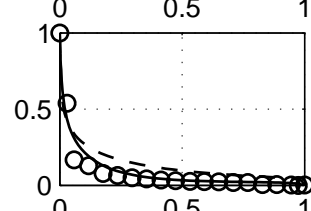

(c)

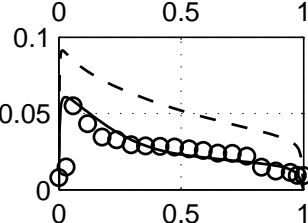

0.1
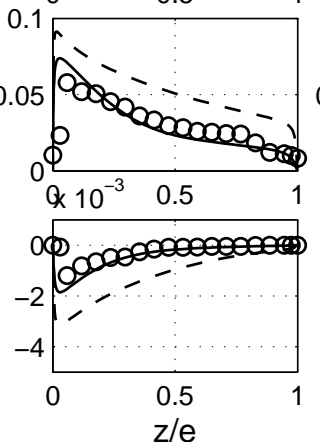

(d)
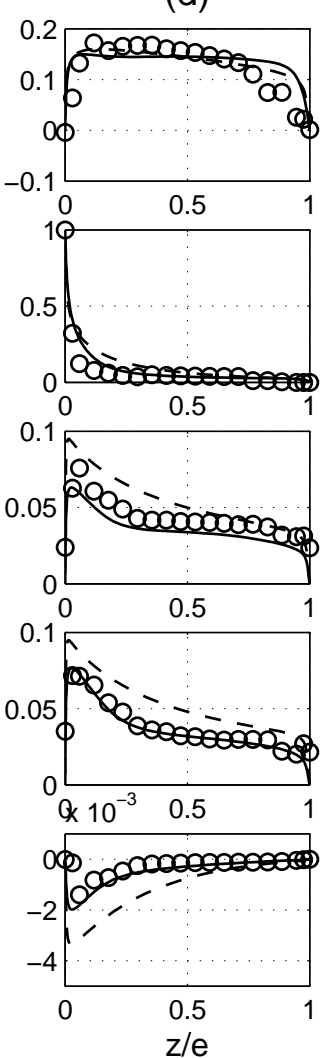

Fig. 4. Poncet et al., submitted to Int. J. Heat Fluid Flow. 


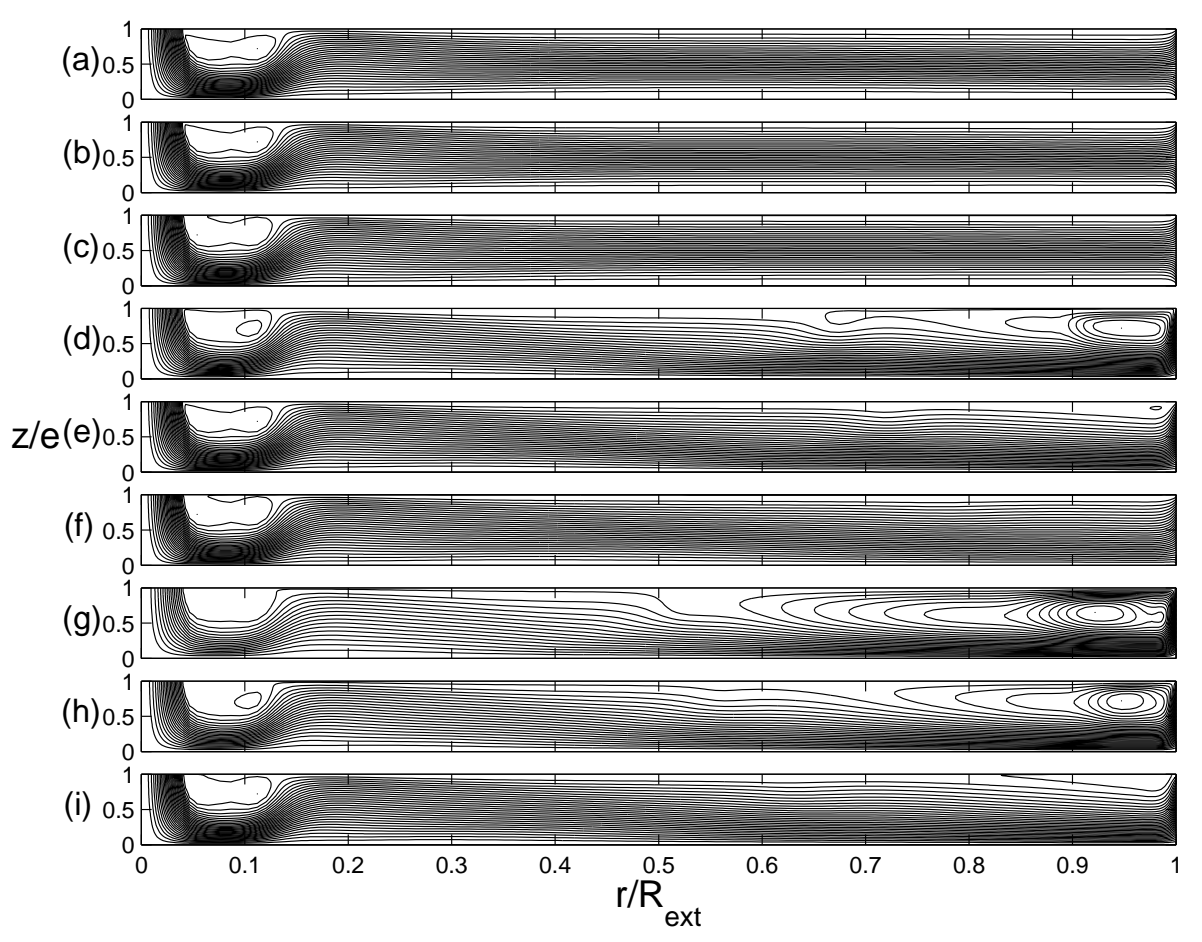

Fig. 5. Poncet et al., submitted to Int. J. Heat Fluid Flow. 
(a)

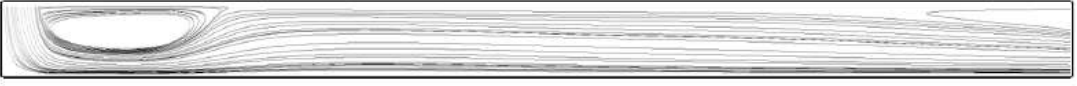

z/e (b)

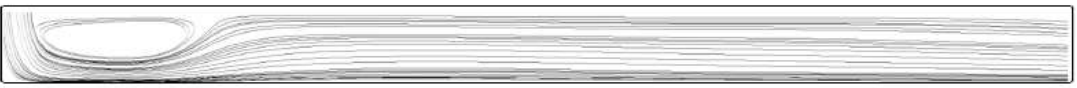

(c)

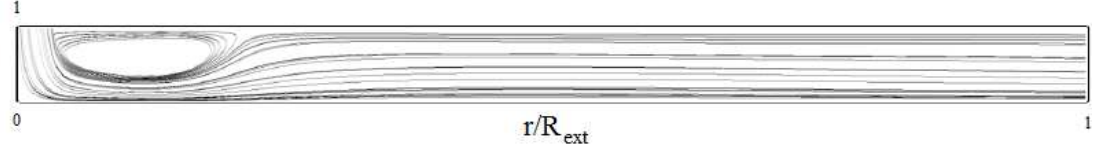

Fig. 6. Poncet et al., submitted to Int. J. Heat Fluid Flow. 
(a)
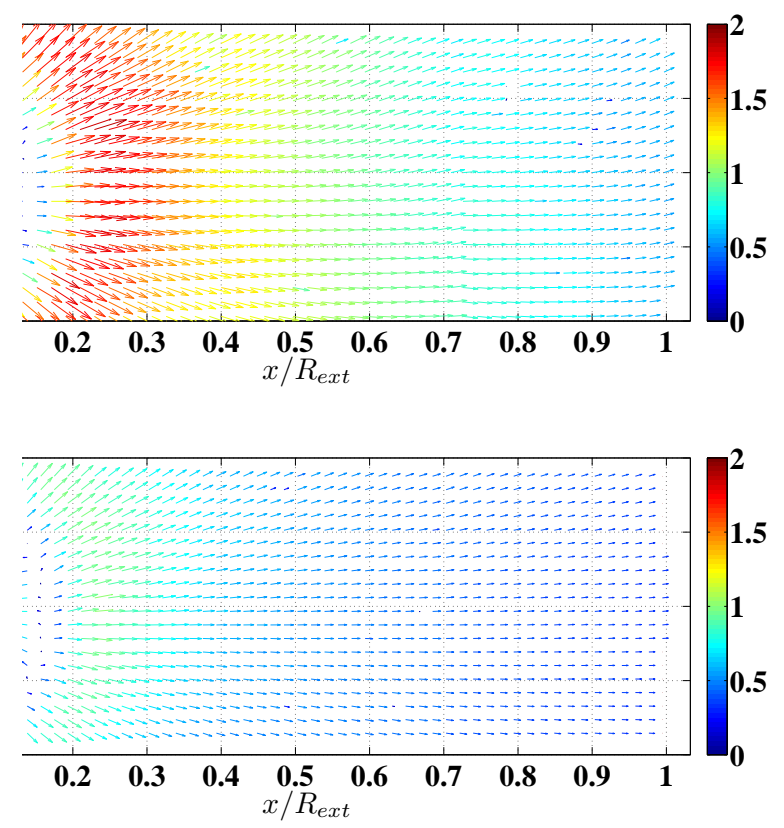

(b)

(c)
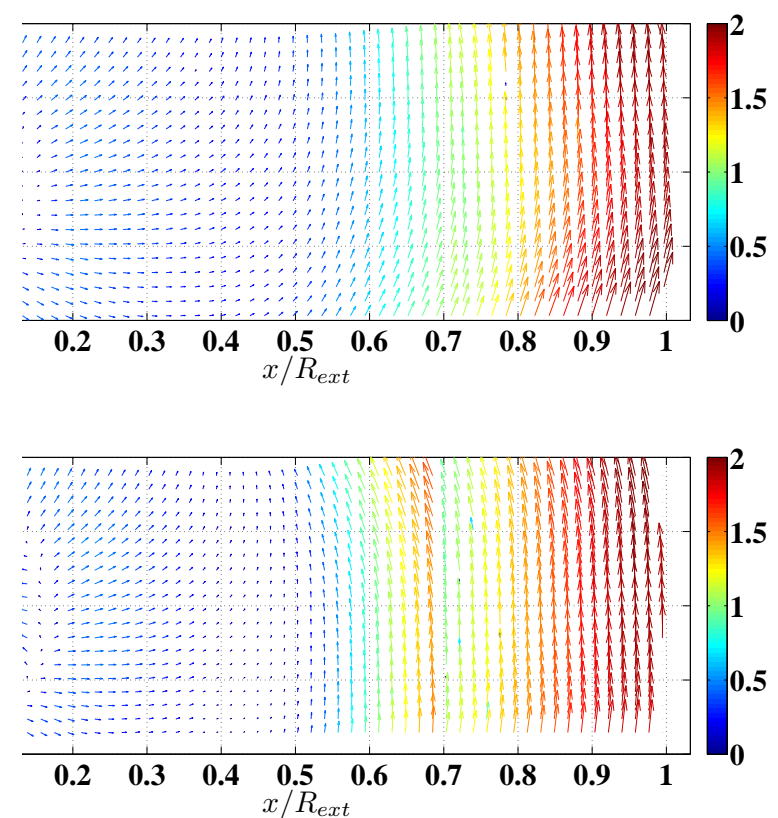

(d)

Fig. 7. Poncet et al., submitted to Int. J. Heat Fluid Flow. 


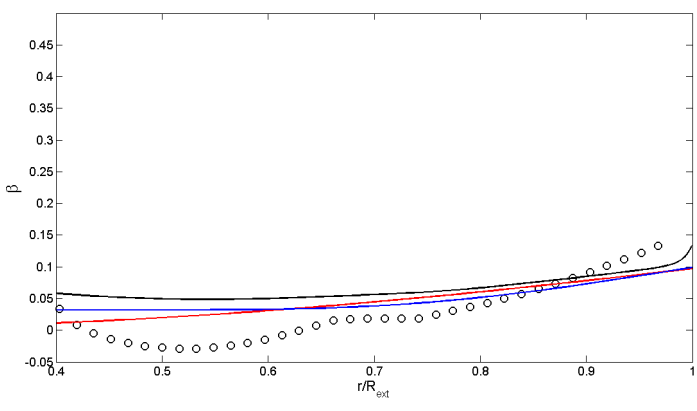

(a)

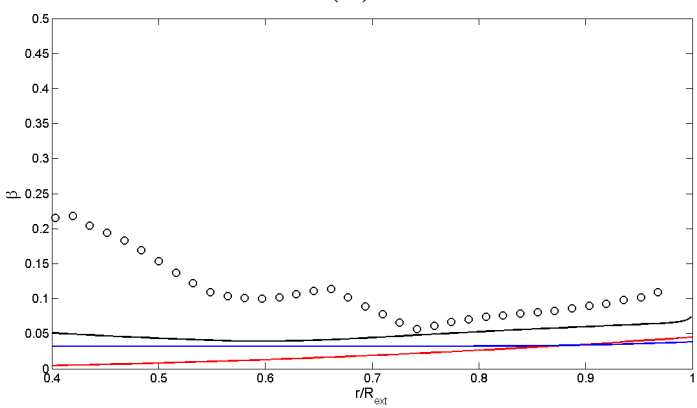

(c)

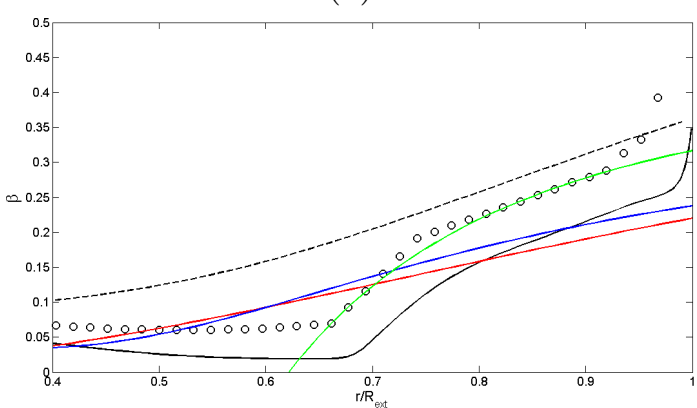

(e)

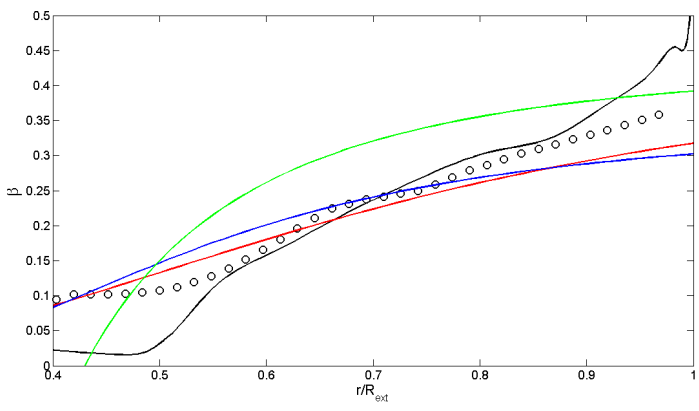

(g)

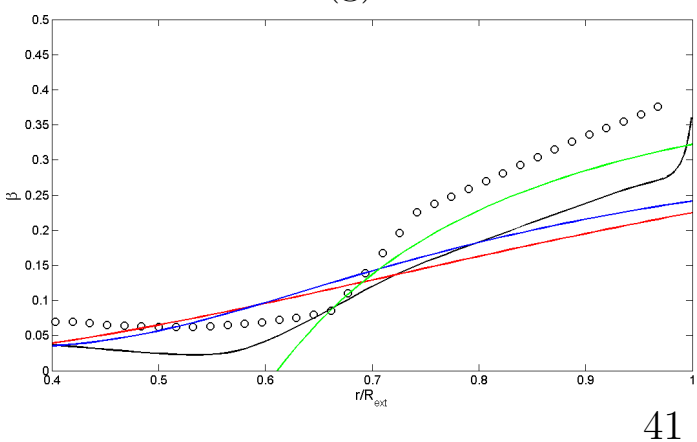

(i)

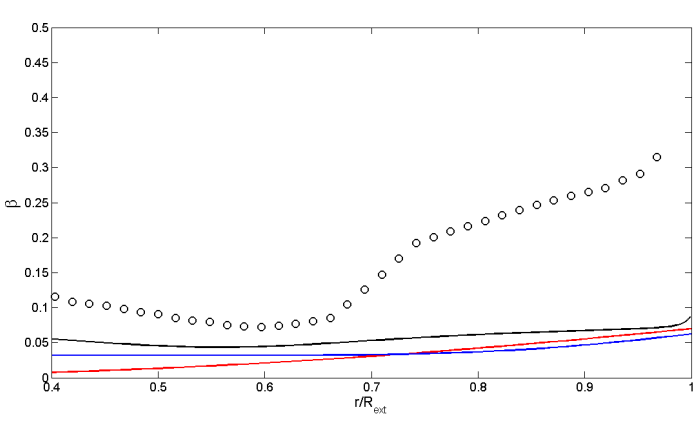

(b)

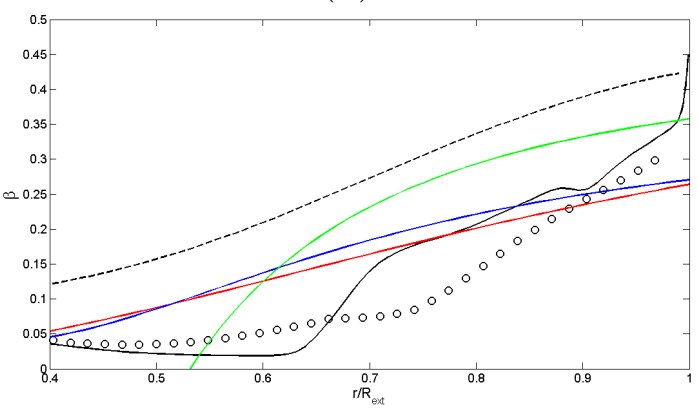

(d)

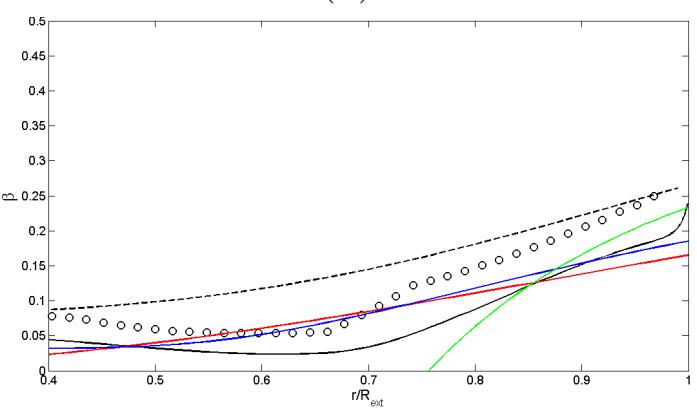

(f)

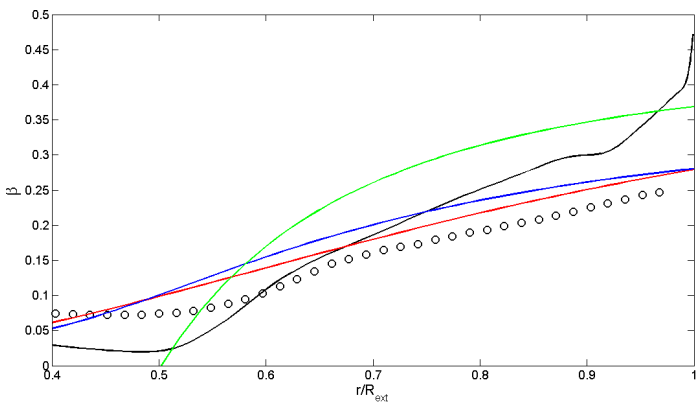

(h)

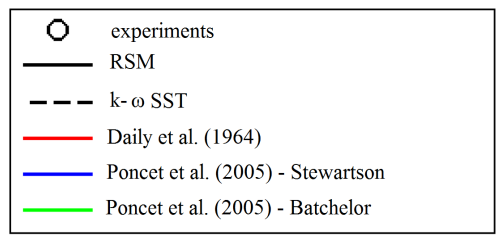

Fig. 8. Poncet et al., submitted to Int. J. Heat Fluid Flow. 


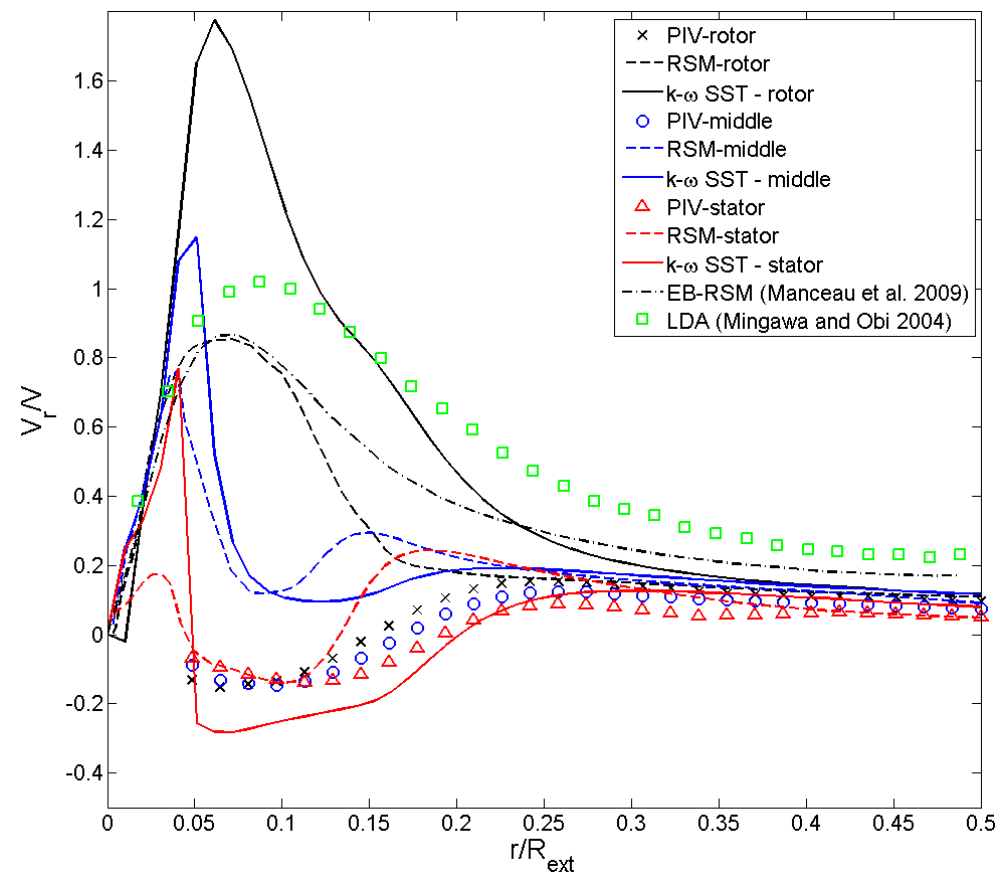

Fig. 9. Poncet et al., submitted to Int. J. Heat Fluid Flow. 
(a)
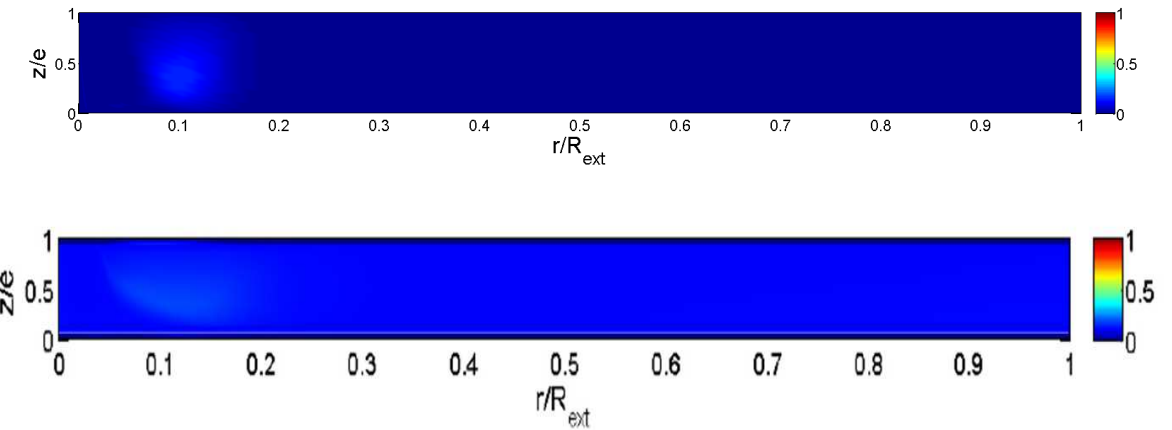

(b)

(c)
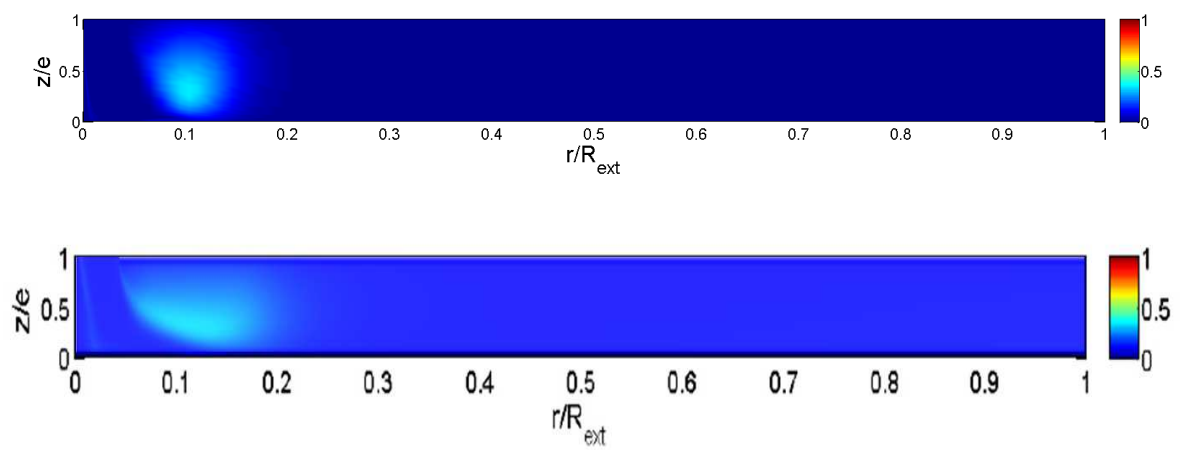

(d)

(e)
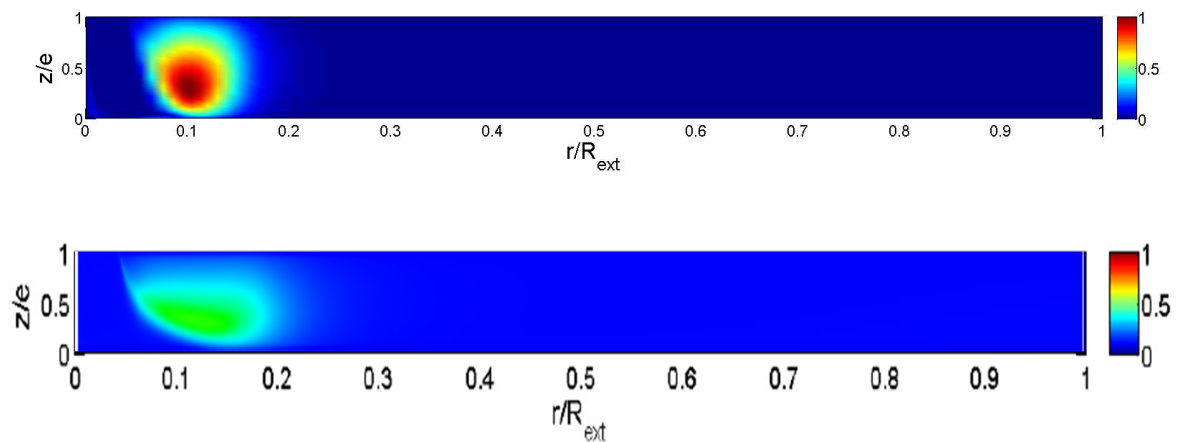

(f)

Fig. 10. Poncet et al., submitted to Int. J. Heat Fluid Flow. 

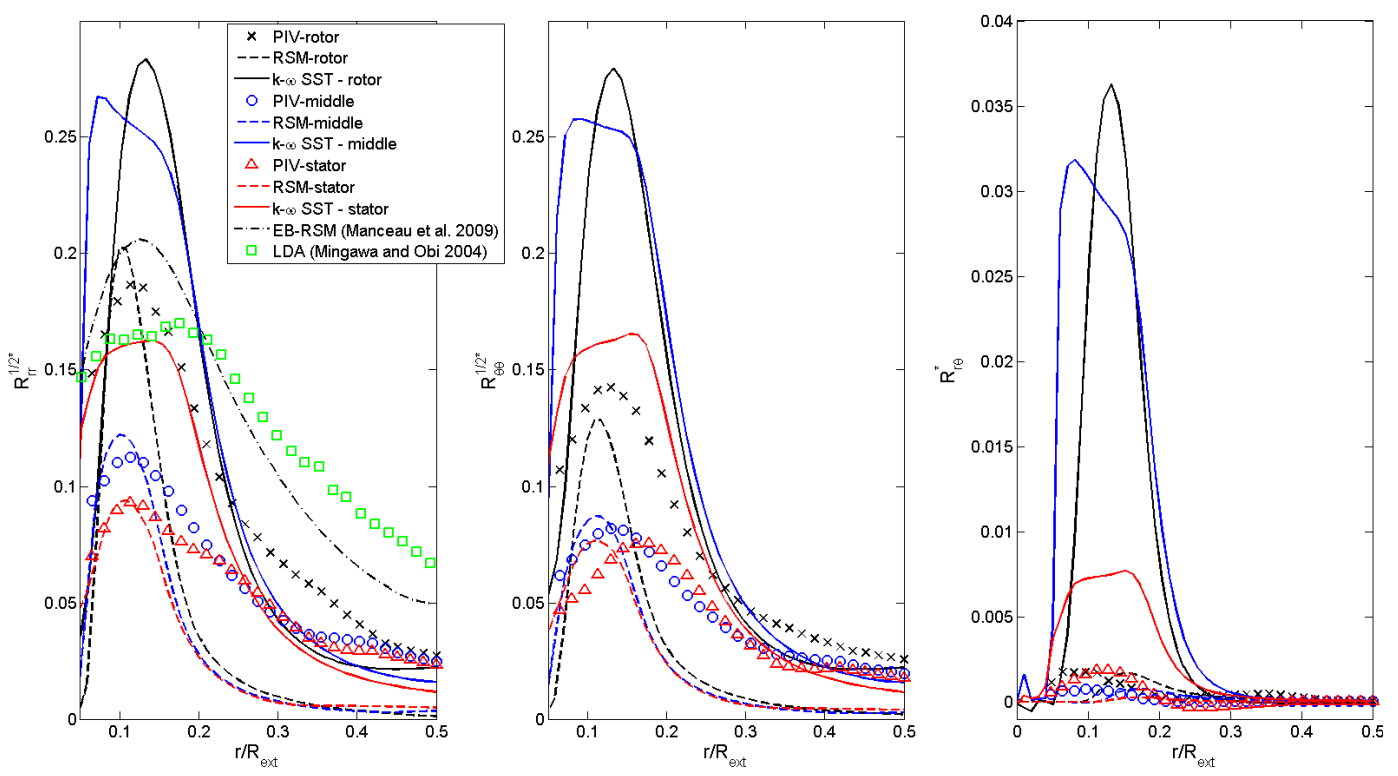

Fig. 11. Poncet et al., submitted to Int. J. Heat Fluid Flow.
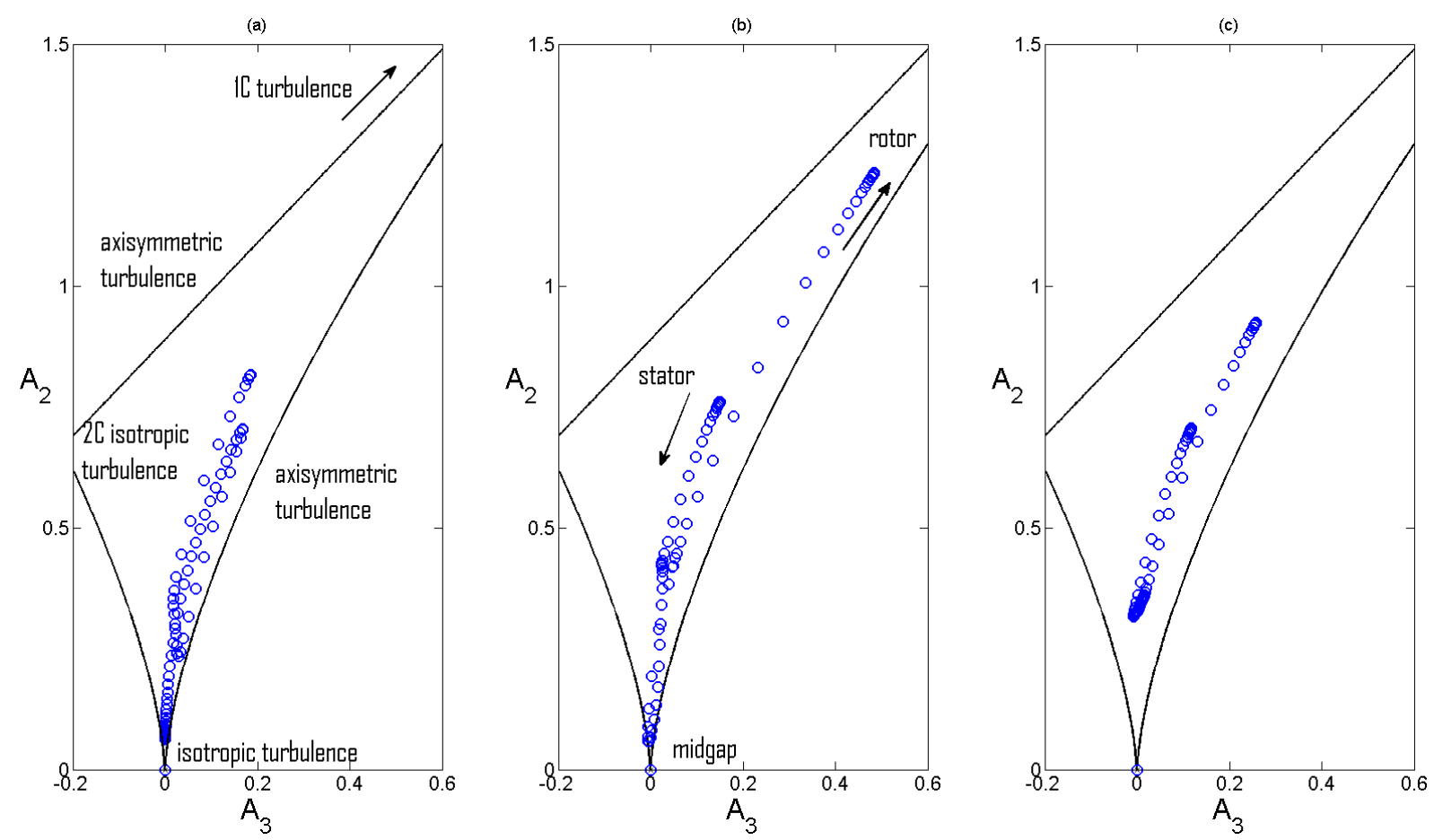

Fig. 12. Poncet et al., submitted to Int. J. Heat Fluid Flow. 
(a)

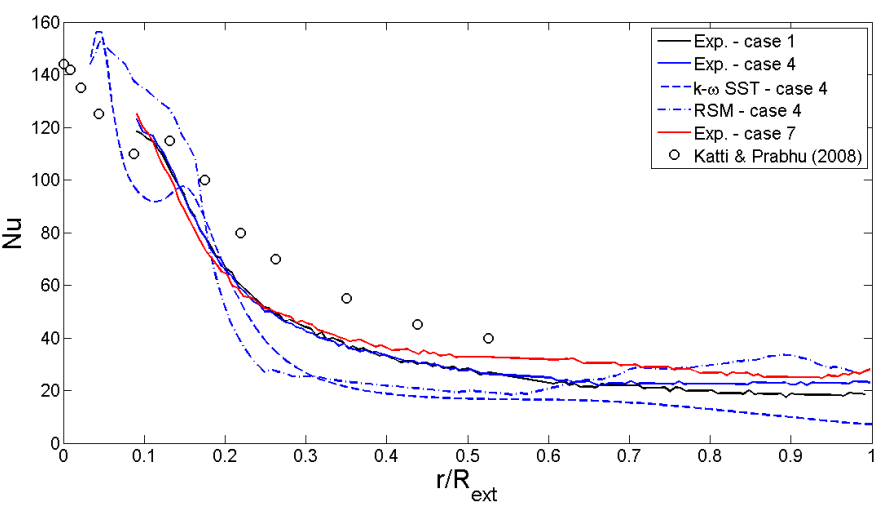

(b)

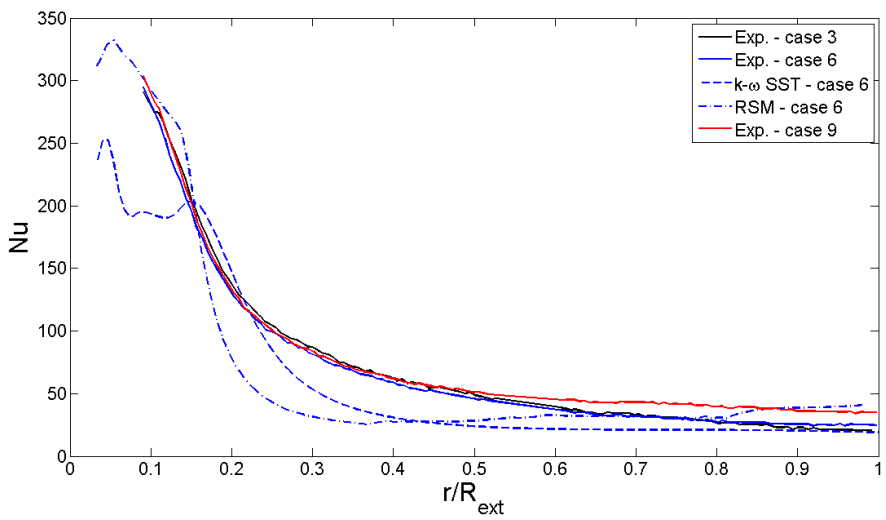

Fig. 13. Poncet et al., submitted to Int. J. Heat Fluid Flow.

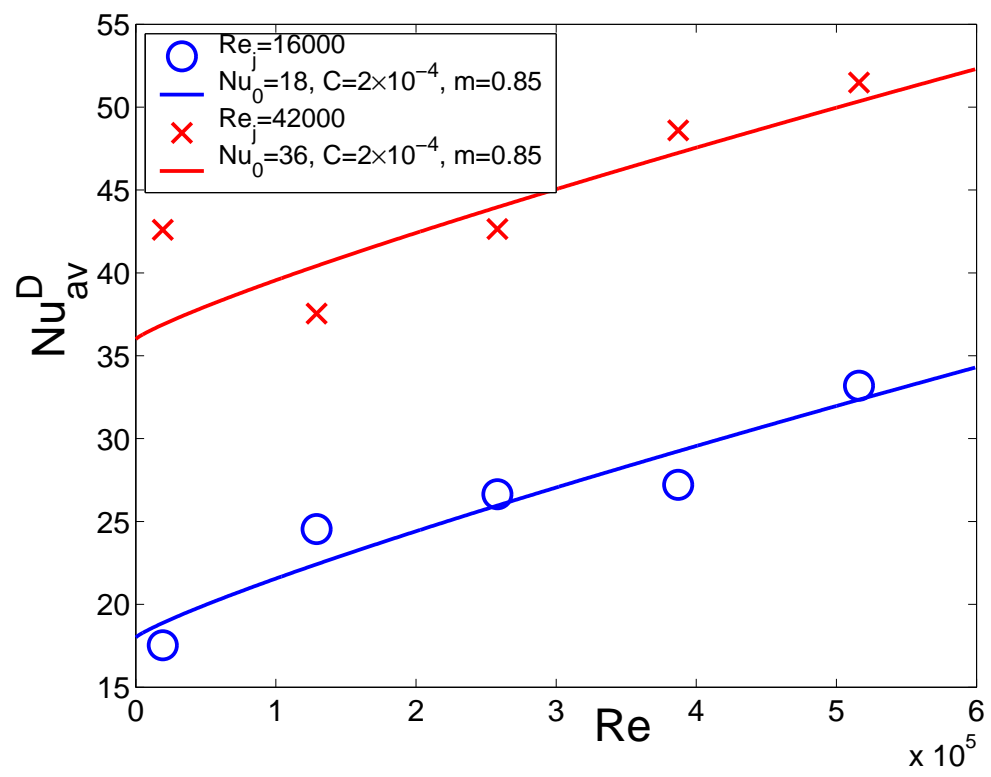

Fig. 14. Poncet et al., submitted to Int. J. Heat Fluid Flow. 